\title{
Erfahrungen aus der Hochschullehre
}

\author{
Inhaltsverzeichnis
}

3.1 Zur Rolle einer sprachbewussten Lehramtsausbildung Mathematik: Ein Gespräch mit Studierenden - 122

3.1.1 Vorstellung der Studienprojekte - 122

3.1.2 Stellenwert des Themas Sprachbildung in Hochschule und Schule - 126

3.1.3 Forschen im Praxissemester - 127

3.1.4 Forschungsfragen finden - 130

3.1.5 Methoden und Inhalte im Seminar - 131

3.2 Deutsche und türkische Schüler*innenlösungen im Vergleich - 132

3.3 Sprachbildung in der Lehramtsausbildung Informatik - 140

3.3.1 Einleitung - 140

3.3.2 Seminarkonzeption - 141

3.3.3 Ausgewählte Aspekte der Fachsprache im Informatikunterricht - 143

3.3.4 Erste Erfahrungen und Ausblick - 149

3.4 Chemische Konzepte und Sprache im Übergang - Ein Seminarkonzept zur praxisnahen Ausbildung von Lehramtsstudierenden in den Fächern Sachunterricht und Chemie in der Sekundarstufe I - 150

3.4.1 Einleitung - 150

3.4.2 Planung von Unterricht - 150

3.4.3 Durchführung und Reflexion von Unterricht - 156

3.4.4 Fazit und Ausblick - 157 


\subsection{Ausblick - 157}

3.5.1 Aktivitäten im Rahmen der Kooperation - 158

3.5.2 Sprachbildung in der Hochschule: Ansatzpunkte zur

Vernetzung - 160 
Die bisherigen Ausführungen thematisieren praktische Erfahrungen mit den Konzepten, die zunächst in $>$ Kap. 1 hergeleitet und theoretisch fundiert und dann in $\triangleright$ Kap. 2 in praxisbezogener Perspektive ausgearbeitet wurden. In diesem Kapitel kommen unterschiedliche Stimmen von Studierenden sowie von Kolleginnen und Kollegen zu Wort.

- Abschn. 3.1 dokumentiert ein Gespräch mit Studierenden, das im Anschluss an die Lehrveranstaltungen geführt wurde, vor deren Hintergrund die in Kap. 1 und 2 entwickelten Konzepte entstanden sind. Teilgenommen an dem Gespräch haben auch ehemalige Studierende, die zum Zeitpunkt des Gespräches bereits ihren Vorbereitungsdienst absolvierten und die Leitideen einer sprachbewussten Lehramtsausbildung im Fach Mathematik vor dem Hintergrund ihrer bisherigen Praxiserfahrungen reflektieren konnten. Den Auftakt für das Gespräch bildet eine Rückschau auf die Studienprojekte, die die Studierenden im Rahmen der Lehrveranstaltungen ausgearbeitet hatten und die zu Beginn zusammenfassend wiedergegeben werden. Im Verlauf des Gespräches diskutieren die Studierenden dann etwa den Stellenwert von Sprachbildung in Hochschule und Schule, das forschende Lernen im Rahmen von Praxisphasen sowie nicht zuletzt die Frage, welche der in den Lehrveranstaltungen thematisierten Inhalte und Methoden für ihre Tätigkeit als Lehrkräfte von Bedeutung sind.

In Abschn. 3.2 werden von Sümeyye Erbay praxisbezogene Erfahrungen mit Elementen einer sprachbewussten Lehramtsausbildung bei der Vorbereitung und Durchführung eines Studienprojekts im Praxissemester aus der Perspektive einer (nunmehr ehemaligen) Studierenden dokumentiert. Inhaltlich wird dabei die Nutzung der Herkunftssprache im Mathematikunterricht am Beispiel des Türkischen adressiert.

Fatma Batur und Jan Strobl berichten in Abschn. 3.3 über ihre Kooperation zwischen der Didaktik der Informatik und dem Projekt „Deutsch als Zweitsprache in allen Fächern (ProDaZ)“ an der Universität Duisburg-Essen zum Thema „Sprachbildung im Informatikunterricht". Die hohe Relevanz dieses Themas zeigt sich vor allem darin, dass Kompetenzen im Bereich der „Digital Literacy“ von größter gesellschaftlicher Bedeutung sind, allerdings noch kaum beforscht ist, wie solche Kompetenzen in geeigneter Weise im Unterricht unter besonderer Berücksichtigung sprachbildender Ansätze vermittelt werden können. Der Beitrag zeigt exemplarisch, welche Anknüpfungspunkte auf curricularer, inhaltlicher und hochschuldidaktischer Ebene die hier beschriebenen Aspekte für andere Fächer bieten.

In Abschn. 3.4 beschreiben Melanie Beese, Dennis Kirstein und Henning Krake ein Seminarkonzept zur praxisnahen Vorbereitung von Lehramtsstudierenden auf sprachsensiblen Unterricht in den Fächern Sachunterricht (Grundschule) und Chemie (HRSGe). Im Mittelpunkt stehen dabei die theoretisch fundierte Planung und praktische Erprobung von Lernsequenzen am Übergang vom naturwissenschaftlichen Sach- zum chemischen Fachunterricht. Berücksichtigt werden dabei sowohl zentrale Qualitätsmerkmale des experimentgestützten Chemieunterrichts (z. B. Kontextorientierung, kognitive Aktivierung) als auch spezifische sprachliche Anforderungen und Fördermöglichkeiten im Fach Chemie.

- Abschn. 3.5 schließlich gibt einen Ausblick mit praktischen Anregungen zur Kooperation und Vernetzung unterschiedlicher fachlicher Domänen - auch und gerade mit Blick auf einen Transfer der hier beschriebenen Konzepte. 


\subsection{Zur Rolle einer sprachbewussten Lehramtsausbildung Mathematik: Ein Gespräch mit Studierenden}

Das Thema „Sprache im Fach Mathematik“ ist aus wissenschaftlicher Perspektive so komplex wie interessant. Ziel der vorliegenden Konzepte und Beispiele für die Gestaltung von Lehrveranstaltungen ist es, Studierenden durch die interdisziplinäre Zusammenarbeit eine Sichtweise zu ermöglichen, bei der fachliche und sprachliche Aspekte im Studium sowie im Unterricht als zusammengehörig wahrgenommen werden. Zugleich soll dem Thema Sprachbildung - wie es eine Studentin während einer Veranstaltung einmal formuliert hat - mehr Leichtigkeit verliehen werden.

Um mit einem gewissen zeitlichen Abstand zu den Lehrveranstaltungen auch Eindrücke aus Studierendensicht zu gewinnen, wurden im November 2019 vier ehemalige Studierende eingeladen, die die Lehrveranstaltungen zur Vorbereitung und Begleitung des Praxissemesters Mathematik bei in den Jahren 2017/18 bzw. 2018/19 besucht hatten.

Das Gespräch war durch Impulse zu folgenden Themenblöcken locker vorstrukturiert:

- Vorstellung der Studienprojekte: Inwiefern sind Aspekte der Verknüpfung von Sprache und Fach in Ihre Studienprojekte mit eingeflossen?

- Stellenwert des Themas Sprachbildung in Hochschule und Schule: Welche Seminarthemen erachten Sie im Rückblick als besonders wichtig?

- Forschen im Praxissemester: Wie beurteilen Sie das Verhältnis von wissenschaftlichen und praxisbezogenen Anteilen im Rahmen des Seminarzyklus?

- Methoden und Inhalte im Seminar: Welche Methoden, welche Seminarinhalte, welche wissenschaftlichen Verfahren „,bleiben“ Ihnen für die Zukunft?

Die Gesprächsbeiträge werden im Folgenden in entsprechenden Abschnitten wiedergegeben. Zum Auftakt stellten die Studierenden die Studienprojekte vor, die sie während des Praxissemesters an den Schulen durchgeführt hatten. Dafür wurden den Studierenden ihre Prüfungshandouts zur Verfügung gestellt.

Wie das (aus Gründen der Lesbarkeit leicht adaptierte) Transkript zeigt, bestritten die Studierenden das Gespräch im Wesentlichen untereinander. Wir danken Isabelle Brachtendorf, Sümeyye Erbay, Nina Pooth und Yasin Türkmenoglu sehr herzlich für ihre Bereitschaft, gemeinsam einen Rückblick auf die Lehrveranstaltungen zu werfen. Bei Julia Stechemesser bedanken wir uns für die Unterstützung bei der Abschrift und Erstellung dieses Kapitels.

\subsubsection{Vorstellung der Studienprojekte}

In diesem Abschnitt werden die Studienprojekte, die die Studierenden im Rahmen der Praxissemestervorbereitung und des Begleitseminars erarbeitet und in der Schule durchgeführt haben, vorgestellt. Die Studierenden erläutern Inhalte und Rahmenbedingungen des Projekts und geben zentrale Ergebnisse wieder. Es schließt sich jeweils eine Reflexion an. 


\section{- Studienprojekt 1: Numerische Aufgaben und Textaufgaben mit unterschiedlichen sprachlichen Hürden}

YT: „Ich habe in meinem Projekt die Mathematikleistung bei Textaufgaben mit unterschiedlichem sprachlichen Anspruchsniveau untersucht. Ich habe den Schülerinnen und Schülern Textaufgaben gegeben, wo bei gleichem Fachinhalt unterschiedlich viele sprachliche Hürden zu finden waren. Zusätzlich zu der jeweiligen Textaufgabe wurden die Aufgaben noch in der numerischen, also entkleideten Form, gelöst. Ich habe im Rahmen einer quantitativen Auswertung die Aufgaben der ganzen Klasse mit einem Punktesystem korrigiert und mir die entsprechenden Ergebnisse notiert. Die Ergebnisse haben gezeigt, dass die numerischen Aufgaben zu über $80 \%$ richtig bearbeitet wurden. Die Aufgaben mit niedrigerem sprachlichen Anspruchsniveau wurden zu $64 \%$ richtig gelöst und die sprachlich schwierigen Aufgaben haben $49 \%$ richtig gelöst. Ich habe dann die interessantesten Lösungen herausgesucht und mit den Schülerinnen und Schülern ein Interview geführt. Am Anfang der Interviews sollten sie erzählen, wie sie die Aufgabe gelöst haben. Dabei konnten sie zuerst frei erzählen und dann habe ich konkretere Fragen gestellt. Ich habe die Schüler auf Fehler aufmerksam gemacht, um ihre Denkprozesse besser verstehen zu können. Bei Aufgaben wie: Lisa geht einkaufen, sie kauft drei Lutscher zu je achtzig Cent und zwei Flaschen Cola zu je einem Euro zehn., wurde deutlich, dass schon einfache Präpositionen wie beispielsweise die Präposition je große Schwierigkeiten verursachen können, wenn sie übersehen werden. Aus diesem Grund habe ich dieses Projekt überhaupt begonnen. Eine Schülerin hatte dieses Wort übersehen, beziehungsweise sie wollte es übersehen, weil sie es nicht verstanden hat. Ich habe daraus gelernt, dass wirklich einfache Wörter, die für uns normal sind, für Lernende, und damit meine ich auch Muttersprachler, große Schwierigkeiten darstellen können. Ich habe mir dann die Frage gestellt, ob ich als angehender Lehrer zum Beispiel im Rahmen von Prozentrechnung, wo Präpositionen eine wichtige Rolle spielen, nur die Mathematikleistung bewerte oder ob ich tatsächlich die sprachlichen Leistungen bewerte. Würden die Schülerinnen und Schüler die entkleidete Aufgabe richtig lösen? Sollte man beim Bewerten Sprache und Mathematik voneinander trennen oder gehört zu der Mathematik das Beherrschen der Fachsprache dazu? Also sind Bewertungen fair, bei denen sprachliche Komponenten gar nicht einbezogen werden? Ist die Aufgabe des Mathelehrers doch nur das Beibringen einer Formel? Ich habe eine Sensibilität dafür gewonnen, dass Fachsprache explizit und an die Zielgruppe angepasst vermittelt werden muss. Ich versuche, in meinem Rahmen und aufgrund der Literatur, die wir im Seminar behandelt haben, mit der Problematik umzugehen. Ich habe bei meinem Projekt die Textaufgaben natürlich vorher durchdacht, aber die Fragen im Interview waren der Dynamik des Gespräches untergeordnet. Ich konnte schon Einblicke in die Prozesse gewinnen, aber beim nächsten Mal würde ich systematischer vorgehen. Eigentlich sind die Interviews das Herzstück des Ganzen, weil ich dort eins zu eins die Prozesse nachvollziehen konnte. Im Nachhinein hätte ich bei den Interviews ein bisschen organisierter sein können. Ich habe sehr offen angefangen, um das Gespräch ein bisschen lockerer zu machen und ich wusste im Interview ja, an welchen Stellen die Schülerinnen und Schüler Schwierigkeiten hatten. Ich habe die Aufgaben gesammelt und konnte mir in den Aufgabenbearbeitungen die Stellen markieren. Dann habe ich versucht, die Fragen konkret auf ein bestimmtes Problem einer Schülerin oder eines Schülers zu beziehen. Vielleicht hätte ich das anders machen sollen. Ich habe auf diese Weise das Gespräch sehr gelenkt; beim nächsten Mal würde ich darauf achten, dass die Fragen systematischer gestellt werden. Die Dynamik sollte aber trotzdem aufrecht erhalten bleiben.“ 


\section{- Studienprojekt 2: Textaufgaben in deutscher und türkischer Sprache}

SE: „Meine Forschungsfrage war: Welche Unterschiede lassen sich bei mehrsprachigen Schülerinnen und Schülern bei der Bearbeitung von Textaufgaben in der Muttersprache und in der deutschen Sprache feststellen? Mein Ziel war es, fachliche und sprachliche Auffälligkeiten von Schülerinnen und Schülern mit Migrationshintergrund zu ermitteln. Ich habe zehn Schülern der 9. Klasse zwei Aufgaben gestellt. Die eine Aufgabe war in deutscher Sprache und die andere Aufgabe war in türkischer Sprache. Ich habe die Schüler gebeten, die Aufgaben in der jeweiligen Sprache zu bearbeiten. Die Aufgaben bezogen sich thematisch auf lineare Gleichungssysteme. Dazu muss ich noch erwähnen, dass der Notendurchschnitt zum Thema lineare Gleichungen in der Klasse nicht gut war. Die Ergebnisse haben gezeigt, dass die Schülerinnen und Schüler sowohl bei der türkischen als auch bei der deutschen Aufgabe Schwierigkeiten hatten, die Textaufgabe zu verstehen und die mathematischen Anforderungen zu erfüllen. Insgesamt hatten sie bei der türkischen Aufgabe größere Schwierigkeiten. Ich glaube, man hat häufig die Vorstellung, dass ein Kind, das zweisprachig aufgewachsen ist, beispielsweise Türkisch besser sprechen und verstehen kann als Deutsch. Bei der Aufgabe war es aber nicht so. Die Schwierigkeiten im Türkischen ergaben sich aus Problemen mit der Bildungssprache. Wenn die Kinder hier aufwachsen, nehmen sie am deutschen Mathematikunterricht teil und Aufgaben werden auf Deutsch bearbeitet. Dementsprechend fiel es den Schülerinnen und Schülern schwer, typische mathematische Begriffe wie beispielsweise Additionsverfahren zu übersetzen. Im Nachhinein hätte ich die Schülerinnen und Schüler gerne interviewt, weil es mich sehr interessiert hätte, warum ihnen die Aufgabenbearbeitung so schwergefallen ist. Fünf von ihnen haben die Aufgabe gar nicht bearbeitet, was ich auch sehr interessant fand.“

\section{- Studienprojekt 3: Sprachliche und mathematische Hürden bei kontextgebundenen Aufgaben}

IB: „Meine Forschungsfrage war: Wie strukturieren Lernende den Prozess der Bearbeitung kontextgebundener Aufgaben und auf welche innermathematischen und sprachlichen Hürden stoßen sie dabei? Ich habe einem Schüler und einer Schülerin der 8. Klasse Blütenaufgaben zu linearen Gleichungen vorgelegt, die immer schwieriger wurden. Die beiden haben sich bei der gemeinsamen Bearbeitung selbst mit dem Smartphone aufgenommen und ich habe das im Anschluss transkribiert. Dann habe ich analysiert, wie sie bei der Lösung der Aufgabe vorgegangen sind und welche sprachlichen und mathematischen Probleme sie hatten. Obwohl sie die Aufgaben ein paar Wochen zuvor im Unterricht gelöst hatten, hatten sie überhaupt keinen Ansatz. Sie wollten die Aufgabe zuerst mithilfe einer Skizze lösen, was nicht funktioniert hat. Dann haben sie es mit Ausprobieren versucht, hatten aber extreme Probleme, sich gegenseitig zu verstehen bei dem, was sie da gemacht haben. Also der eine hat das gesagt, der andere hat das dann aber nicht verstanden und sie haben versucht, zusammen zu arbeiten, aber das war eben auf sprachlicher Ebene relativ schwierig. Durch das Projekt habe ich gelernt, dass man Vorgehensweisen mit Schülerinnen und Schülern intensiv üben muss. Und mir ist bewusst geworden, wie sprachintensiv Mathematik eigentlich ist. Es ist außerdem wichtig, dass Schülerinnen und Schüler Strategien verinnerlichen. Beim nächsten Mal würde ich den Schülerinnen und Schülern nicht so viele Aufgaben geben. Ich habe ihnen viele arithmetische Aufgaben gegeben und hatte dann im Nachhinein das Problem, 
eine Forschungsfrage zu finden, die dazu passte. Ich bin dabei, glaube ich, etwas untypisch vorgegangen, weil ich mir die genaue Forschungsfrage erst im Nachhinein überlegt und hundert Mal umgeworfen habe. Ich hätte die anderen Aufgaben einfach weglassen können und mich nur auf die Textaufgaben fokussieren können.“

\section{- Studienprojekt 4: Vergleiche mit relativer Häufigkeit in der sechsten Klasse}

NP: „In meinem Projekt ging es um das Thema relative Häufigkeit. In der sechsten Klasse wurden den Schülerinnen und Schülern Prozentzahlen beziehungsweise Brüche und Zusammenhänge erklärt. Ich wollte, dass den Schülerinnen und Schülern bewusst wird, warum dieses Thema wichtig ist. Ich habe dann überlegt, dafür die relative Häufigkeit zu nutzen. Ich glaube, es ist generell wichtig, das Warum gleich am Anfang zu klären. Meine Forschungsfrage lautet: Inwiefern können Schülerinnen und Schüler der Klasse 6 einen Vergleich mit Hilfe der relativen Häufigkeit anstellen, ohne diese explizit erlernt zu haben? Inwiefern sind sie in der Lage, ihre Ergebnisse zu versprachlichen? Dafür habe ich zuerst eine Aufgabe konzipiert, die sich durch die Auswahl des Themas Fortnite an den Interessen der Schülerinnen und Schüler orientiert hat. Ich habe dann Aussagen konzipiert, die die Schüler als richtig oder falsch bewerten sollten. Dazu habe ich ihnen Formulierungshilfen gegeben. In einer weiteren Aufgabe wurden dann die relativen Häufigkeiten von zwei Klassen verglichen, wodurch ich letztendlich meine Forschungsfrage beantworten konnte. Gerade im Fach Mathematik gab es in dieser Klasse viele Defizite. Für die Argumentationen haben wir mit Scaffolding gearbeitet. Die Ergebnisse haben gezeigt, dass die acht Schülerinnen und Schüler, die bisher eine Klasse wiederholen mussten oder große Probleme im Fach Mathematik hatten, auch die Aufgaben nicht beantwortet haben. Am Ende konnte ich sagen: Ja, es ist möglich, diesen Vergleich über die relative Häufigkeit bereits in der sechsten Klasse anzustellen und nicht, wie der Lehrplan es vorgibt, in der achten oder neunten Klasse. Das liegt daran, dass in der Klasse die Lernvoraussetzungen und das Vorwissen über Brüche und Prozentzahlen gegeben waren. Meine Ergebnisse haben dem Leistungsniveau der Klasse entsprochen. Bezüglich der Frage, ob die Schülerinnen und Schüler in der Lage waren, das zu versprachlichen, bin ich zu dem Ergebnis gekommen, dass es in diesem Bereich große Probleme gab. Ich habe durch das Studienprojekt natürlich gemerkt, dass Sprache in der Mathematik eine Rolle spielt. Ich habe auch unabhängig von meinem Projekt erfahren, wie häufig Scaffolding eingesetzt wird und dass Versprachlichungsmöglichkeiten mittlerweile tatsächlich in den Unterricht gelangen. Insgesamt denke ich, Textaufgaben sollten nicht zu sehr vereinfacht werden, damit man den Schülerinnen und Schülern nicht die Möglichkeit nimmt, daran zu wachsen. Auf der anderen Seite sollen sie aber auch nicht mit zu schwierigen Aufgaben überfordert werden. Es geht darum, eine Mitte zu finden. Man kann dann sagen: Ok, diese Aufgabe ist zu schwer und ich mache die ein bisschen leichter. Die Präposition je lasse ich in der Aufgabe und erkläre sie im Unterricht. Man kann den Schülerinnen und Schülern dadurch die Möglichkeit geben, daran zu wachsen, und das war für mich eine wichtige Erkenntnis. Wenn ich dieses Projekt noch einmal durchführen würde, würde ich eine ähnlich komplexe Problemstellung wählen. Ich würde die Aufgabenstellung präziser und simpler gestalten, direkt mehr Möglichkeiten des Scaffoldings einbinden sowie Differenzierung ermöglichen, beispielsweise in Form von Blütenaufgaben. Ich würde kleinschrittiger vorgehen und für das Projekt einen längeren Zeitraum einplanen.“ 


\subsubsection{Stellenwert des Themas Sprachbildung in Hochschule und Schule}

Im folgenden Gesprächsausschnitt geht es um den Stellenwert von Sprachbildung im Lehramtsstudium allgemein sowie das Verhältnis von Mathematikdidaktik und Sprache in den Lehrveranstaltungen. Es wird deutlich, dass die Studierenden den konkreten Fachbezug für wichtig halten. Mit Blick auf die Schulpraxis zeigt sich, dass die Studierenden ein Bewusstsein für das Thema „Sprache im Fach“ entwickelt haben und auch konkrete Umsetzungsmöglichkeiten im Unterricht kennen.

\section{- Gesprächsausschnitt 1, Sprachbildung in Hochschule und Schule}

IB Ich fand das ganz gut an unserem Seminar, dass wir von Anfang an einen roten Faden hatten, der sich durchzog, was die Thematik anging: dass es immer um Sprache und Mathematik ging und nicht irgendwie so eine bunte Mischung aus allem Möglichen war, das nicht miteinander in Verbindung steht.

SE Ja, das finde ich auch. Ich finde es gut, dass es diese Angebote gibt, bei denen ein Fach mit Deutsch als Zweitsprache kooperiert. Allerdings ist es möglich, dass Studierende mit dem Thema Sprachsensibilität nicht in Berührung kommen, wenn sie diese Seminare nicht bewusst wählen. Es wäre wünschenswert, dass sich alle Studierenden mit diesem Thema auseinandersetzen müssen.

NP Wir kommen ja durch das DaZ-Modul allgemein mit Sprachbildung in Berührung, aber eben nicht spezifisch in Verbindung mit dem Fach Mathematik. Ich finde, es ist schon wichtig, dass man den ganz klaren Bezug zu seinem Fach gewinnt.

YT Wir sind ja alle Sek-I-Studierende, ne? Die Uni ist ja dafür zuständig, uns Wissen zu vermitteln, das wir im Unterrichtsalltag verwenden können. Und ich denke, es ist eine Verweigerung der Realität, wenn man das System in dem Sinne tricksen kann, dass man einfach andere Seminare wählt. Also um es auf den Punkt zu bringen: Ich glaube nicht, dass es irgendeinen Mathematikunterricht gibt, in dem man keine sprachförderlichen Elemente braucht. Das Thema Sprachförderung ist im Studium meiner Meinung nach immer noch zu wenig vertreten. Und ich glaube, dass viele Kollegen und Kolleginnen in der Schule mit der Situation überfordert sind. Hier in diesem Seminar habe ich für mich persönlich die Chance gehabt, Empathie aufzubauen. Ich meine, ich habe sowieso erst die türkische Sprache erlernt, danach die deutsche; für mich ist das Thema sowieso schon sensibel. Solche Seminare können eine Empathie aufbauen und haben das ja auch getan. Ich erinnere mich noch daran, dass wir im Seminar eine Aufgabe auf Englisch oder auf einer Sprache, die wir nach der Deutschen am besten können, bearbeiten sollten.

Dabei merkt man erst, welche Probleme Schülerinnen und Schüler haben, die vor Aufgaben in einer Sprache gestellt werden, die sie nicht oder nicht so gut verstehen. Ich finde es für angehende Lehrer und Lehrerinnen wichtig, eine Sensibilität zu entwickeln.

SG Wie haben Sie das Verhältnis von Sprache und Mathematikdidaktik in dem Seminar empfunden?

IB Ich fand das Verhältnis von Sprache und Mathe eigentlich sehr ausgewogen. Wir hatten davor relativ viele Seminare, die sich ausschließlich mit Mathematik befasst haben. Daher fand ich es erfrischend, eine andere Perspektive einzunehmen, die sonst zu kurz kommt. 
NP Sprache hatte im Seminar die ganze Zeit „,beiläufig“ oder „nebenbei“ einen gewissen Stellenwert. Das ist ja stellvertretend dafür, wie es später auch in unserem Unterricht sein soll. Und ich finde, das kann man sehr gut übertragen.

SG Sie haben im letzten Seminar über eine gewisse Leichtigkeit in Bezug auf das Thema Sprachbildung und Mathematikunterricht gesprochen.

NP Ja, das stimmt. Im Praxissemester habe ich zum Beispiel eine Lehrerin kennengelernt, die viele Fortbildungen zum Thema Sprachbildung im Fach Mathematik besucht und viel neuen Input geben kann. Sie integriert Sprachförderung in den Unterricht, indem sie bei den Schülern einfach mal nachfragt: „Wie meinst du das denn genau?“" oder „Erklär doch mal, wie bist du da vorgegangen bist.“ Und das meine ich mit ,Leichtigkeit": Dass es gar nicht so schwer ist, dass man keinen Riesenberg vor sich sehen muss als Student oder als Lehrer. Dass man eben schon mit Kleinigkeiten etwas bewirken kann. Man muss sich nicht für jede Unterrichtsstunde lange auf einen sprachsensiblen Umgang vorbereiten. Es geht mehr darum, spontan im Unterricht eine Chance zu ergreifen. Und was das Seminar vielleicht gerade gezeigt hat: Dass es ja manchmal schon reicht, wenn man kleine Worte in einer Aufgabenstellung bespricht oder kleine Veränderungen vornimmt und damit die Schüler schon sprachlich unterstützen kann.

YT Ich wollte noch einen allgemeineren Punkt anmerken. Die Situation im Praxissemester ist in Bezug auf die zeitlichen Ressourcen, die einer Lehrkraft zur Verfügung stehen, ein bisschen künstlich. Im schulischen Alltag wird man nie die Möglichkeit haben, sich so intensiv mit einer Klasse zu beschäftigen. Daher würde ich mich dem anschließen, was vorhin gesagt wurde: Durch solche interdisziplinären Seminare entwickelt man einfach so ein Bewusstsein für bestimmte oder für potenzielle Hürden und man sorgt mit kleinen Hilfen dafür, dass die Kinder lernen, diese Hürden zu bewältigen.

\subsubsection{Forschen im Praxissemester}

In diesem Gesprächsausschnitt geht es um die Bedeutung forschenden Lernens im Praxissemester. Einerseits wird der Sinn des forschenden Lernens mit Blick auf die spätere Unterrichtstätigkeit in Frage gestellt. Andererseits verdeutlichen die Studierenden, dass sie durch die intensive Auseinandersetzung im Studienprojekt neue Perspektiven einnehmen und theoretische Ansätze besser auf die Schulpraxis beziehen können. Das forschende Lernen wird zudem als Chance gesehen, um eigene Hypothesen zu überprüfen und verschiedene Methoden auszuprobieren.

\section{- Gesprächsausschnitt 2, Forschen im Praxissemester}

NP Wir sind ja alle Lehramtsstudenten. Wir möchten alle in die Schule gehen und Wissen vermitteln - aber keiner von uns hat sich ja dafür entschieden zu forschen. Und auf einmal wird uns abverlangt, dass wir Forschungsfragen entwickeln. Normalerweise liefert man im Studium einfach ab. Man fragt sich gar nicht mehr, was einen selbst interessiert, was man selbst erforschen möchte. Forschung ist keine Richtung, in die man selbst gehen möchte. Ich finde, das driftet von dem eigentlichen Ziel ab, aber vielleicht ist das auch ein falscher Blickwinkel.

SE Ich verstehe, was du meinst. Ich habe mich im Praxissemester auch gefragt, warum wir das machen. 
NP Ja, warum sollen wir forschen? Natürlich werfen sich im Praxissemester Fragen auf, aber die Formulierung dieser Fragen und das Finden von Antworten ist so weit von dem entfernt, wo wir eigentlich hinmöchten. Es ist eine andere Richtung als die, die wir eigentlich eingeschlagen haben.

IB Ja, ich finde aber, dass das schon auch von der Frage abhängt, der du auf den Grund gehst. Manche Fragestellungen können dich für deine spätere Berufstätigkeit schon weiterbringen.

NP Ja, das stimmt, man kann mal einen anderen Blickwinkel auf die Sachen werfen, und es steht nicht immer nur die reine Wissensvermittlung im Vordergrund.

IB Ja, zum Beispiel auch die Arbeit mit Audios oder Videos, die eben angesprochen wurde.

SE Ich glaube, da ist es wichtig, dass das Thema einen einfach interessiert, ne?

NP Genau, ich möchte nicht in Frage stellen, dass es einen weiterbringen kann. Nur diesen Schritt des wissenschaftlichen Aufbereitens von Fragen und dann die Auswertung der Daten, das ist...

IB Hast du das so empfunden? Ich muss zugeben, ich hatte nie das Gefühl, dass ich so richtig wissenschaftlich arbeite, weil ja auch alles in einem so kleinen Rahmen war.

FS Vielleicht machen wir das nochmal konkret. Frau P., Sie hatten zum Beispiel die Forschungsfrage: Inwiefern können Schülerinnen und Schüler der sechsten Klasse einen Vergleich mit Hilfe der relativen Häufigkeiten anstellen, ohne dies vorher explizit thematisiert zu haben und inwiefern sind sie in der Lage ihre Ergebnisse zu versprachlichen? Wenn Sie diese Forschungsfrage jetzt rückblickend betrachten, haben Sie eine Antwort auf Ihre Forschungsfrage gefunden?

NP Ja, natürlich, und sich sage ja auch nicht, dass das alles keinen Sinn ergibt. Es hat sich zum Beispiel herausgestellt, dass Inhalte, die eigentlich nach dem Lehrplan erst später vermittelt werden sollten, schon früher verwendet werden können. Das war eine Erkenntnis, die ich ja nicht nur für dieses spezielle Thema, sondern generell für mich gewonnen habe.

YT Also ich sehe das Ganze so: Jeder weiß ja, wie man theoretisch ein Auto fährt, aber solange man nicht selbst gefahren ist, kann man das Auto auch nicht fahren. Ein Beispiel: Aus der Literatur wusste ich natürlich, dass es sprachliche Hürden gibt. Aber erst als ich im Rahmen des Studienprojekts den Kontakt zu den Schülerinnen und Schülern hatte und mit ihnen darüber geredet habe und ihre Sichtweise reflektiert habe, ist mir das Problem so richtig bewusst geworden. Deswegen finde ich es sehr gut, dass die Möglichkeit besteht, das auszuprobieren, bevor man in den Lehreralltag einsteigt.

IB Ja, dann kann man auch über den Tellerrand hinausschauen.

YT Also, dass man experimentiert, in diesem Sinne.

IB Also man kann im Studium auch mal etwas machen, was vielleicht nicht unbedingt direkt etwas mit dem Berufsleben zu tun hat.

YT Ich glaube, wenn man später im Referendariat und dann im Berufsalltag ist, ist man nicht mehr so offen gegenüber neuen Sachen. Man ist so sehr von Dingen gestresst und fragt sich, wie man das Ganze bewältigen soll, anstatt nach neuen Methoden zu suchen. Das war auch die Resonanz von Kollegen, mit denen ich darüber gesprochen habe. Man sollte als angehender Lehrer beziehungsweise als angehende Lehrerin offen sein für das Lesen wissenschaftlicher Bücher und die Auseinandersetzung mit der Wissenschaft. Ich habe im Praxissemester bemerkt, 
dass viele Personen an der Schule die Ansätze aus dem Bereich Deutsch als Zweitsprache, die in der Literatur beschrieben werden, nicht kennen. Und manchmal wurde Fleiß und nicht die Sprache als Erklärung benutzt, warum ein Schüler oder eine Schülerin eine Sache versteht oder nicht. Aus diesem Grund sollte der wissenschaftliche Anteil an der Universität stärker fokussiert werden als zum Beispiel praktische Aspekte wie die Unterrichtsplanung. Wenn eine Lehrkraft Prozesse und Faktoren, die im Lernprozess wichtig sind, nicht versteht, ist selbst die beste Unterrichtsplanung nicht hilfreich.

SE Ich finde, das eine schließt das andere nicht aus. Wenn ich zum Beispiel Unterricht planen würde und Deutsch als Zweitsprache wäre ein Bestandteil, dann wäre ja auch die Sprachsensibilität ein entscheidender Faktor darin, oder?

YT Ja genau, ich wollte nur noch mal auf die Kritik eingehen, dass das Praxissemester zu wissenschaftlich ist. Ich empfinde den universitären Teil des Praxissemesters nicht als zu wissenschaftlich. Ich denke, es ist eine Chance für die Studierenden, so zu arbeiten. Das schließt nicht aus, dass ich im Berufsalltag noch weiter forsche, ich habe hier ja die Methoden kennengelernt. In Bezug auf ältere Lehrkräfte möchte ich auch nicht pauschalisieren. Sie haben aber häufig weniger Kontakte zur Universität aufbauen können und in Gesprächen zum Thema Sprachsensibilität kristallisiert sich schnell heraus, wie Ursachen für Probleme geschildert werden. Ich denke, dass die Seminare insbesondere im Fach Mathematik eine gute Möglichkeit bieten, in einem reflektierten Raum Wissen vermittelt zu bekommen und forschen zu können.

FS ... was natürlich genau das ist, was wir uns fürs Praxissemester wünschen: Dass Sie als Studierende die Zeit haben, in einem geschützten Rahmen etwas auszuprobieren und das zusammen in der Universität theoretisch zu reflektieren und zu vertiefen. Das ist so das Idealbild des forschenden Lernens, wo Theorie und Praxis wirklich zusammenkommen.

SG Und die Idee dahinter ist natürlich, dass Sie das später auch anwenden können. Wenn Sie das einmal gemacht haben und gemerkt haben: „Ok, ich habe wirklich eine Antwort dadurch bekommen“, können Sie auch später als Lehrer oder Lehrerin ein Problem mit der Klasse wissenschaftlich angehen, also Daten sammeln, systematisch anschauen und eventuell etwas am Unterricht verändern. Das ist der große Bereich der Lehrerhandlungsforschung oder Aktionsforschung. Wir haben natürlich die Hoffnung, dass Sie durch das forschende Lernen auch in Ihrer Lehrtätigkeit später objektiver an Probleme herangehen können.

NP Ich glaube, die Sätze, die Sie gerade formuliert haben, hätten mir zu Beginn des Seminars geholfen. Es wäre für mich logischer gewesen, wenn ich die Ziele, dass man später als Lehrkraft forschen können soll und dass Probleme dadurch objektiver bewältigt werden können, direkt verstanden hätte. Ich würde diese Ziele zu Beginn eines Seminars transparent machen. Das wäre die optimale Antwort auf die Frage: „Warum sind wir hier?“

FS Diese Diskrepanz wird ja häufig erlebt: „Wir sitzen hier und wissen gar nicht, was wir tun sollen und denken uns irgendwelche Studienprojekte aus, die nichts mit der Realität zu tun haben.“

NP Ich glaube, den Sinn versteht man oft erst im Nachhinein. Ich hatte heute noch ein Reflexionsgespräch in meinem anderen Fach. Als ich mir mein eigenes Studienprojekt nochmal durchgelesen habe, habe ich erst so richtig begriffen, was das für 
mich tatsächlich bedeutet. Manchmal hat man erst Wochen oder Monate später so ein Aha-Erlebnis. Wenn das Ziel vorher vorgegeben wird, steigert das vielleicht die Motivation, Inhalte zu erarbeiten.

\subsubsection{Forschungsfragen finden}

Im folgenden Interviewausschnitt geht es um das Thema „Finden einer Forschungsfrage“, das die Studierenden im Kontext des Praxissemesters als besonders wichtig empfinden. Sie gehen insbesondere auf die Schwierigkeit ein, aus einem individuellen Interesse heraus eine geeignete Forschungsfrage zu entwickeln. Als hilfreich wird der Austausch in der Seminargruppe, aber auch die Unterstützung durch die Dozierenden eingeschätzt. Letzteres ist gerade an einer solch sensiblen wie wichtigen Stelle im Forschungsprozess (dem Finden von Forschungsfragen) auch inhaltlich nachvollziehbar, wenngleich das Ziel solcher forschenden Lernprozesse natürlich darin besteht, Studierende zu selbständigem wissenschaftlichen Arbeiten anzuleiten.

\section{- Gesprächsausschnitt 3, Forschungsfragen finden}

FS Welche Themen haben Sie in Bezug auf das Studienprojekt im Praxissemester als besonders wichtig empfunden?

NP Für mich war eines der wichtigsten Themen im Seminar, wie man eine Forschungsfrage findet. Damit tue ich mich furchtbar schwer. Bei mir haben sich super viele Forschungsfragen aufgeworfen, die aber dann alle doch nicht Hand und Fuß hatten. Dazu gehörte zum Beispiel die Frage, wie man Frustration im Fach Mathematik vorbeugen kann, oder welche Methoden sich eignen, um die Angst vor dem Fach Mathematik zu reduzieren. Nur darauf zu achten, welche Fragen einen wirklich interessieren, ist vielleicht nicht der einzige Weg, eine Forschungsfrage zu finden.

YT Bei mir resultierte die Forschungsfrage aus der Frage, wie ich, insbesondere in Bezug auf die Sprache, mit den bestehenden Problemen umgehen würde. Dass die Mathematik mit ihren Textaufgaben auch für Muttersprachler sprachliche Hürden bereithält, kann ja niemand abstreiten. Aber wie gehen Lehrer damit um? Wie würde ich damit umgehen? Das war sozusagen meine Motivation, und daraus habe ich dann eine Forschungsfrage entwickelt.

FS Wenn wir die Projekte in ihrer Entwicklung betrachtet haben, ist uns aufgefallen, dass bei vielen Studierenden häufig solche Ausgangsfragen da waren: Wie ist die Motivation im Mathematikunterricht? Wie ist die sprachliche Entwicklung? Wie kann ich bestimmte Dinge fördern? Und dass diese Fragen im Verlauf immer spezifischer wurden. Können Sie das auf Ihren eigenen Prozess anwenden oder war das bei Ihnen nicht so?

NP Ja, doch, innerhalb des Seminars, das stimmt. Ich formuliere es vielleicht einmal anders: Innerhalb des Seminars bin ich gut unterstützt worden, sodass ich die Formulierung einer Forschungsfrage geschafft habe. Trotzdem fände ich aber auch eine Hilfestellung wichtig, die mich beim selbstständigen Finden einer Forschungsfrage unterstützt.

FS Und was waren Punkte, die Sie als Unterstützung empfunden haben?

NP Die Nachfragen der Dozenten ... 
IB ... und der Austausch im Seminar. Wenn man alleine zuhause ist und überlegt, worüber man ein Projekt machen kann, ist das schwierig. Dass wir uns im Seminar ausgetauscht haben, dass wir uns die Projekte gegenseitig vorgestellt und Schwächen und Stärken herausgearbeitet haben: Das fand ich hilfreich. Das ist eine gute Vorbereitung. Aber du hast recht, der Austausch mit den Dozenten ist da nochmal ein bisschen ...

NP ... expertenhaltiger, ja.

IB Es ist schön, wenn jemand dabei ist, der die Thematik besser überblicken kann.

SE Ja, ich weiß noch genau, wie ich vor meinen Daten saß und dachte: Was hab' ich denn da herausgefunden? Ich dachte, ich habe gar nichts herausgefunden, was spannend ist. Da hat mir die individuelle Beratung schon sehr weitergeholfen.

SG Könnten Sie vier sich, wo Sie diesen Prozess schon einmal durchlaufen haben, bei einem neuen Problem besser helfen?

IB Ich glaube schon, aber ad hoc fände ich das schwierig, ich bräuchte dafür länger Zeit. Wenn ich zuhause in Ruhe darüber nachdenken würde und auch ein bisschen Zeit hätte, etwas zu recherchieren, dann könnte ich besser Hilfestellung geben.

\subsubsection{Methoden und Inhalte im Seminar}

Abschließend wird über die Methoden und Inhalte aus dem Seminar gesprochen, die den Studierenden besonders in Erinnerung geblieben sind und die sie als besonders wichtig für ihre spätere Tätigkeit als Lehrkräfte einschätzen. Als Beispiele werden forschungsmethodische Aspekte (z. B. Audio-/Videoanalyse), das Thema der sprachbewussten Aufgabenvariation sowie die eigene Sensibilisierung für die Bedeutung von Sprache im Fachunterricht genannt.

\section{- Gesprächsausschnitt 4, Methoden und Inhalte im Seminar}

SG Was bleibt Ihnen besonders im Gedächtnis von dem, was wir mit Ihnen in den zwei Semestern erarbeitet haben und was Sie selbst im Studienprojekt erarbeitet haben? Welche Inhalte und Methoden können Sie auf Ihren aktuellen Unterricht beziehen, wenn Sie schon in der Schule tätig sind? Gibt es wissenschaftliche Verfahren, die Sie anwenden können, um Ihre Schüler besser zu verstehen?

IB Für mich persönlich war es super spannend, die Schüler aufzunehmen und diese Aufnahme dann zu analysieren. Dadurch konnte ich ihre Herangehensweisen bei der Aufgabenbearbeitung nachvollziehen. Im Unterricht sieht man ja meistens hinterher nur das Ergebnis und kann den Denkprozess, der im Kopf stattfindet, nicht nachvollziehen. Ich könnte mir vorstellen, das später in Einzelfällen auch mal im Unterricht einzusetzen. Ich würde die Schüler bitten, ihre Denkprozesse aufzuschreiben und zu erklären oder bei der Partnerarbeit eine Audioaufnahme zu machen. Es ist zwar immer so eine Sache mit dem Datenschutz, aber grundsätzlich ist es ja kein großer Mehraufwand. Also das fand ich super spannend und da wäre ich vorher vielleicht gar nicht so auf die Idee gekommen.

NP Ich fand das Thema Aufgabengestaltung und Aufgabenvariation super spannend. Mir sind in Mathebüchern schon oft Aufgaben aufgefallen, die nicht gut formuliert und für die Schüler unmotivierend waren. Jetzt habe ich einen kleinen Methodenpool, wie ich Aufgaben mit einfachen Mitteln selbst umgestalten oder 
umstrukturieren kann. Das fand ich mega spannend, weil ich damals genau zu den Schülern gehört habe, die große Probleme mit Textaufgaben hatten - auch wenn ich ja Muttersprachlerin bin. Ich finde es auch wichtig, eine Aufgabe so zu gestalten, dass die Schüler sie interessant finden. Bei vielen Aufgaben geht es um Themen wie beispielsweise Bakterien, wo die Schüler sagen: Das interessiert mich einfach nicht. Als Lehrkraft kann man auch einfach mal so nett sein und eine Aufgabe anpassen: Erstens, wie gesagt, um die Schüler fachlich und sprachlich zu fördern, gleichzeitig aber auch, um sie zu motivieren. Das fand ich sehr interessant.

SE Da kann ich euch zustimmen. Im Praxissemester konnte ich das Lehrerhandeln reflektieren und sagen: Ok, das würde ich jetzt anders machen. Ich würde die Aufgabenstellung öffnen oder komplett umformulieren. Wenn ich jetzt teilweise noch Arbeitsblätter für Fünftklässler sehe, kann ich mir echt nicht vorstellen, dass einige Lehrer einfach gar nicht darüber nachdenken, auch wenn an der Schule viele sprachlich schwache Schüler sind. Aber genau das nehme ich auf jeden Fall mit: Ich habe so eine Sensibilität bekommen und das finde ich ganz schön und deswegen war dieses Seminar auch wichtig. Und ich fände es einfach gut bzw. eigentlich eher selbstverständlich, wenn jeder Student damit in Berührung kommt.

YT Was mir aus dem Seminar besonders in Erinnerung geblieben ist: Einmal sollten wir eine Aufgabe in englischer Sprache oder in einer anderen Zweitsprache erklären. Da wurde mir wirklich zum ersten Mal bewusst, dass ich in meiner vermeintlichen Muttersprache [d.h. im Türkischen] gar keine mathematischen Begriffe in diesem Sinne kenne und dass somit die Sprache der Mathematik eigentlich für jeden eine Zweitsprache darstellt, die eigene Strukturen und Ansprüche hat. Mir ist auch in Erinnerung geblieben, dass ich es schwierig fand, die Probleme von Schülern zu lokalisieren. Das ist ein komplexer Prozess, denn die Schwierigkeiten können von mehreren Faktoren abhängig sein. Wir haben in dem Seminar eine Videoaufnahme gesehen von einem Interview mit zwei Schülern. Daran kann man sich orientieren und ich kann mir vorstellen, diese Methode anzuwenden, wenn ein Schüler oder eine Schülerin Probleme hat. Ich würde dann wieder mit Aufgaben arbeiten oder Interviews durchführen, um herauszufinden, wo die Schwierigkeiten wirklich liegen. Das könnte dann vielleicht Einfluss darauf haben, dass ein Schüler oder eine Schülerin erfolgreich wird.

\subsection{Deutsche und türkische Schüler*innenlösungen im Vergleich}

Sümeyye Erbay

Der vorliegende Beitrag thematisiert ein Studienprojekt, welches im Rahmen des Praxissemesters 2018 an einer Realschule im Fach Mathematik mit Schüler*innen der neunten Jahrgangsstufe durchgeführt wurde. Dabei wurde folgende Fragestellung fokussiert: Welche Unterschiede lassen sich bei mehrsprachigen Schüler*innen bei der Bearbeitung von Textaufgaben in der Herkunftssprache und in der deutschen Sprache feststellen?

Das Ziel dieses Projektes war es, sowohl inhaltliche als auch sprachliche Besonderheiten bei den Bearbeitungen der Schüler*innen zu ermitteln. Um die Bearbeitung von Textaufgaben in der Herkunftssprache (in dem Fall Türkisch) und in der deutschen Sprache genauer zu untersuchen, haben die Schüler*innen zwei Aufgaben schriftlich bearbeitet. Hierfür wurden folgende vergleichbare Aufgaben auf Deutsch und Türkisch formuliert, die die Schüler*innen in der jeweiligen Sprache bearbeiten sollten. 


\section{Zwei vergleichbare Aufgaben auf Deutsch und Türkisch (mit deutscher Übersetzung) zur Bearbeitung durch die Schüler*innen}

1) Familie Müller will sich für den Urlaub ein Wohnmobil leihen. Sie bekommt zwei Angebote:

A: Grundgebühr $240 €$ und für jeden Tag der Nutzung $12 €$.

B: Grundgebühr $128 €$ und für jeden Tag der Nutzung $20 €$.

a) Gib die Funktionsgleichung für beide Angebote an. Bestimme vorher, was $x$ und $y$ bedeuten.

b) Stelle beide Funktionsgleichungen grafisch dar. Was bedeutet der Schnittpunkt der beiden Geraden?

c) Prüfe rechnerisch nach, ob du in (b) genau gezeichnet und den Schnittpunkt richtig abgelesen hast. Wende dafür ein dir bekanntes Lösungsverfahren an. Benenne erst, welches Verfahren du anwendest.

d) Welches Angebot sollte Familie Müller nutzen, wenn sie für 3 Wochen in den Urlaub fahren will? Begründe ausführlich (in mindestens 2 Sätzen).

2) $10 \mathrm{~b}$ sınıfı mezuniyet için tişörtlerini mümkün olduğunca ucuza satın almak istiyorlar. Bunun için yapmaları gereken sadece iki teklif arasında seçim yapmaktır.

1. Teklif: Bir baskılı tişört $15 €$ ve tek seferlik işlem ücreti $18 €$.

2. Teklif: Internet üzerinden aldıkları teklifte ise bir tişört $13 €$ 'ya ve ayrıca tek seferlik baskı ve teslimat için $70 €$ hesaplanıyor.

a) Her iki teklifin fonksiyonel denklemini kur ve ayrıca $x$ 'i ve $y$ 'yi belirle.

b) Ikinci teklifin hangi andan itibaren avantajlı olduğunu hesapla. Çözmek için bildiğin bir yöntemi kullan ve açıkla.

c) 10b sınıfında 13 erkek ve 16 kız bulunmaktadır. Yaptığın fonksiyonu göz önünde bulundurarak hangi teklifi almaları gerekir? Açıkla.

d) Mezuniyet tişörtlerin ücretini hesapla.

Die deutsche Übersetzung der zweiten Aufgabe lautet wie folgt:

2) Die Klasse 10b möchte ihre Abschluss-Shirts so günstig wie möglich kaufen. Sie müssen sich lediglich zwischen zwei verschiedenen Angeboten entscheiden.

Angebot 1: Ein bedrucktes T-Shirt kostet $15 €$ und die Bearbeitungsgebühr beträgt einmalig $18 €$.

Angebot 2: Dieses Online-Angebot berechnet pro T-Shirt $13 €$ und für die Druck- und Lieferkosten einmalig $70 €$.

a) Stelle für jedes Angebot eine Funktionsgleichung auf. Bestimme vorher, was $x$ und $y$ bedeuten.

b) Bestimme rechnerisch, ab wieviel T-Shirts das Angebot 2 günstiger ist. Benutze dafür ein dir bekanntes Lösungsverfahren. Benenne erst, welches Verfahren du anwendest.

c) In der Klasse 10b sind 13 Jungs und 16 Mädchen. Für welches Angebot sollte sich die Klasse entscheiden? Begründe.

d) Berechne die Kosten für die Abschluss-Shirts. 
Für die Erstellung dieser Aufgaben wurden zunächst verschiedene Aufgaben aus deutschen Lehrbüchern betrachtet und als Grundlage zur Konzipierung herangezogen. Der inhaltliche Fokus dieses Forschungsvorhabens lag auf funktionalen Aspekten, konkret in Verbindung mit dem Erstellen und Lösen linearer Gleichungssysteme. Die Unterrichtsreihe zum Thema Lineare Gleichungssysteme war zu diesem Zeitpunkt in der Klasse abgeschlossen, wodurch angenommen werden konnte, dass die befragten Schüler*innen Grundkenntnisse zu diesem Teilgebiet erworben hatten.

Im Folgenden werden nun zunächst die fachlichen und sprachlichen Anforderungen dargelegt, die sich aus den Aufgabenstellungen ergeben. Im Rahmen der ersten Teilaufgabe (1a, 2a) sollten die Schüler*innen zwei zu den Vorgaben passende Funktionsgleichungen aufstellen sowie die Variablen x und y definieren. Hierfür sind Kenntnisse über die allgemeine Form linearer Funktionen sowie deren Eigenschaften, wie z. B. über die (un-) abhängigen Variablen, erforderlich. Die sprachliche Anforderung besteht hierbei darin, die Informationen richtig zu deuten, um sie anschließend in die passenden mathematischen Gleichungen zu überführen. Im Rahmen der ersten Aufgabenstellung wurden die Schüler*innen aufgefordert, die von ihnen aufgestellten Funktionsgleichungen in einem Koordinatensystem darzustellen und den Schnittpunkt der beiden Geraden zu interpretieren. Anhand dieser Teilaufgabe (1b) sollte bestimmt werden, inwieweit die befragten Schüler*innen einen Wechsel von der symbolischen zur ikonischen Darstellung vollziehen können. Daraufhin sollten die Schüler*innen das Ergebnis des Gleichungssystems mithilfe eines Lösungsverfahrens bestimmen, um die Lösungsmenge mit dem Schnittpunkt der Funktionsgraphen zu vergleichen. Im Unterschied dazu wird in der zweiten (auf Türkisch formulierten) Aufgabenstellung dieser Darstellungswechsel nicht gezielt gefordert. Stattdessen sollten die Schüler*innen lediglich die Lösungsmenge des Gleichungssystems bestimmen. In beiden Fällen soll das genutzte Lösungsverfahren angegeben werden. Dies setzt nicht nur Kenntnisse über die Bezeichnungen der verschiedenen Verfahren voraus, sondern auch Erfahrungen im Umgang damit. Im letzten Schritt sollten die Schüler*innen bei der Bearbeitung der Aufgabenstellungen eine Aussage über das kosteneffizientere Angebot treffen und die damit verbundenen Kosten rechnerisch bestimmen. Hierbei wurde von den Schüler*innen eine Begründung für die Wahl des Angebots gefordert, die in mindestens zwei Sätzen dargelegt werden sollte. Beide Aufgabenstellungen haben überwiegend die gleichen fachlichen Anforderungen. Der einzige Unterschied besteht darin, dass in der ersten (auf Deutsch formulierten) Aufgabenstellung ein Darstellungswechsel gefordert wird. Neben diesen fachspezifischen Merkmalen stellen die Aufgaben selbst die Schüler*innen vor die sprachliche Herausforderung, Mathematikaufgaben in zwei Sprachen zu verstehen und zu bearbeiten.

Die Stichprobengröße dieser Studie umfasste 10 Schüler*innen einer neunten Klasse, die sowohl die deutsche als auch die türkische Sprache erlernt haben. Alle bis auf einen der befragten Schüler*innen gaben an, dass sie an herkunftssprachlichem Unterricht in der Primarstufe teilgenommen haben. Die Teilnahme konnte aufgrund der nicht vorhandenen schulischen Ressourcen in der Sekundarstufe nicht fortgeführt werden. Der türkische Sprachgebrauch ist im Alltag der Schüler*innen jedoch vorzufinden, da sie neben dem Deutschen auch die türkische Sprache als Kommunikationsmittel im familiären und sozialen Kontext nutzen, sodass sich ein domänenspezifischer Sprachgebrauch zeigt (vgl. Backus 2013, S. 774 ff.).

Für die Bearbeitung der zwei Aufgaben hatten die Schüler*innen insgesamt zwei Unterrichtsstunden Zeit. Die hoch angesetzte Bearbeitungszeit sollte ihnen genügend Zeit zur Verfügung stellen, sich mit beiden Aufgaben intensiv beschäftigen zu können. 
Hospitationen im Unterricht haben gezeigt, dass die Schüler*innen Schwierigkeiten mit Termumformungen sowie bei der symbolischen Darstellung der aufgestellten Funktionsterme hatten.

Mit Blick auf die sprachliche Dimension wurde angenommen, dass die Bearbeitung der Mathematikaufgabe in der Herkunftssprache aufgrund der „,sich längerfristig etablierenden Kultur der Deutschsprachigkeit des Mathematikunterrichts“ (Meyer und Prediger 2011, S. 191) eine größere Herausforderung darstellen würde. Zudem wurde vermutet, dass in den schriftlichen Produkten der Schüler*innen Nonstandardvarianten des Türkischen auftreten, da viele mehrsprachig aufwachsende Schüler*innen in Deutschland durch die fehlende oder unzureichende Vermittlung der türkischen Bildungssprache, wie sie in der Türkei in Bildungsinstitutionen gebraucht wird, keinen sicheren Umgang mit der formalen Sprache erreichen (vgl. Dirim 2009, S. 140 f.). Hingegen wurde bei der Bearbeitung der deutschen Aufgabenstellung aufgrund der Vertrautheit mit ähnlichen Aufgabenformaten aus dem Mathematikunterricht angenommen, dass die Schüler*innen diese Aufgabe besser verstehen würden und dass ihnen die Bearbeitung leichter fallen würde. Hinsichtlich der Textprodukte wurden vor allem Fehlerquellen in den Bereichen der Grammatik und der Lexik erwartet, die für bilingual aufwachsende Schüler*innen typische Herausforderungen darstellen können (vgl. Verboom 2012, S. 14).

Im Folgenden werden Fallbeispiele aufgezeigt und Ergebnisse bezüglich fachlicher und sprachlicher Auffälligkeiten dargelegt, wobei die Bearbeitungen derjenigen Schüler*innen zur Datenauswertung herangezogen werden, die beide Aufgaben vollständig oder zumindest in Ansätzen bearbeiteten. Insgesamt haben fünf der befragten Schüler*innen ausschließlich die erste Aufgabenstellung bearbeitet; diese Bearbeitungen werden daher nicht in der Ergebnisdarstellung aufgegriffen.

Es werden zunächst fachliche Auffälligkeiten dargelegt. Diese wurden mithilfe von Analysekategorien klassifiziert, die anhand der fachlichen Anforderungen der einzelnen Teilaufgaben bestimmt wurden. Folgende Kategorien wurden zur Datenauswertung herangezogen: Aufstellen der Funktionsgleichungen, Zeichnen der Funktionsgraphen, Interpretation des Schnittpunktes der Graphen sowie das Lösen des Gleichungssystems mithilfe eines Lösungsverfahrens.

Die fachliche Analyse der Lernendendaten hat ergeben, dass die Schüler*innen bei der graphischen Darstellung des funktionalen Zusammenhangs z. T. große inhaltliche Schwierigkeiten hatten. Anhand dieser Teilaufgabe sollte ermittelt werden, inwieweit die Schüler*innen einen Darstellungswechsel vollziehen können. Die Aufgabenbearbeitungen zeigen auf, dass der Wechsel von der symbolischen zur ikonischen Ebene häufig eine große Herausforderung für die befragten Schüler*innen darstellte. Einige Schüler*innen konnten die Informationen in den Aufgabenstellungen richtig deuten und die mathematische Anforderung, zwei passende Funktionsgleichungen aufzustellen, erfüllen. Sie besitzen somit Kenntnisse über die allgemeine Form sowie Eigenschaften von Gleichungssystemen, benötigen in der Deutung von Darstellungen und im Darstellungsvernetzungsprozess jedoch Unterstützung. Dies soll im Folgenden anhand eines Beispiels in - Abb. 3.1 verdeutlicht werden. Die Schülerin gibt zunächst beide Funktionsgleichungen an.

Im nächsten Schritt löst sie mithilfe des Subtraktionsverfahrens das Gleichungssystem, obwohl dies erst in Teilaufgabe c gefordert war. Hierin zeigt sich ein gewohnheitsmäßiger, prozeduraler Lösungsautomatismus, der auf formaler Ebene abläuft und das inhaltliche Denken und Deuten der Funktionen umfasst. Sie wendet das Verfahren korrekt 
- Abb. 3.1 Schülerdokument „Lineares Gleichungssystem“ (Aufgabe 1a)

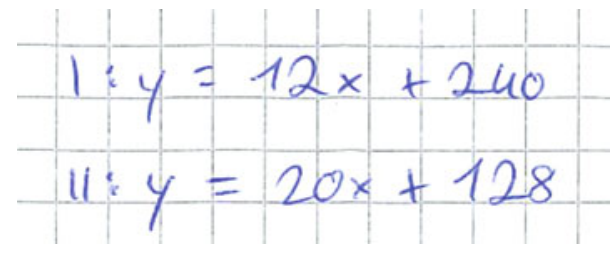

an und bestimmt die richtige Lösungsmenge. Anschließend zeichnet sie den in $\mathbf{0}$ Abb. 3.2 abgebildeten Funktionsgraphen.

Die Vorgehensweise der Schülerin bei der Erstellung dieser Funktionsgraphen kann aufgrund der fehlenden Dokumentation nicht rekonstruiert werden. Es kann lediglich erfasst werden, dass die gezeichneten Funktionsgraphen nicht zu den von ihr aufgestellten Funktionsgleichungen passen.

Insgesamt kann die Schülerin den mathematischen Zusammenhang symbolisch abbilden, ihn jedoch nicht auf die ikonische Ebene übertragen. Im Rahmen ihrer Empfehlung bzgl. des besseren Angebots der Wohnmobilvermietung bezieht sich die Schülerin auf die Funktionsgraphen und gibt an, dass Angebot 2 die kosteneffizientere Variante für den angegebenen Zeitraum darstellt. Es wird aus ihrer Dokumentation jedoch nicht ersichtlich, auf welchen Funktionsgraphen sie sich bezieht. Entgegen ihrer zuvor korrekt berechneten Lösungsmenge deutet die Schülerin die Lösung des Gleichungssystems nicht und bezieht sich am Ende ausschließlich auf die Funktionsgraphen.

Ein weiterer Fokus dieses Projektes lag darin, sprachliche Auffälligkeiten bei der Bearbeitung der beiden Textaufgaben zu ermitteln. Die Analyse der schriftlichen Ausführungen der Schüler*innen ergab, dass Grammatik und Rechtschreibung sowohl im Deutschen als auch im Türkischen nur unzureichend beachtet wurden. Bei der Beschreibung der Herangehensweise für den Aufgabenteil 1b beschrieb eine Schülerin ihre Vorgehensweise wie in $\boldsymbol{\bullet}$ Abb. 3.3 abgebildet.

- Abb. 3.2 Schülerdokument „Funktionsgraphen“ (Aufgabe 1b)

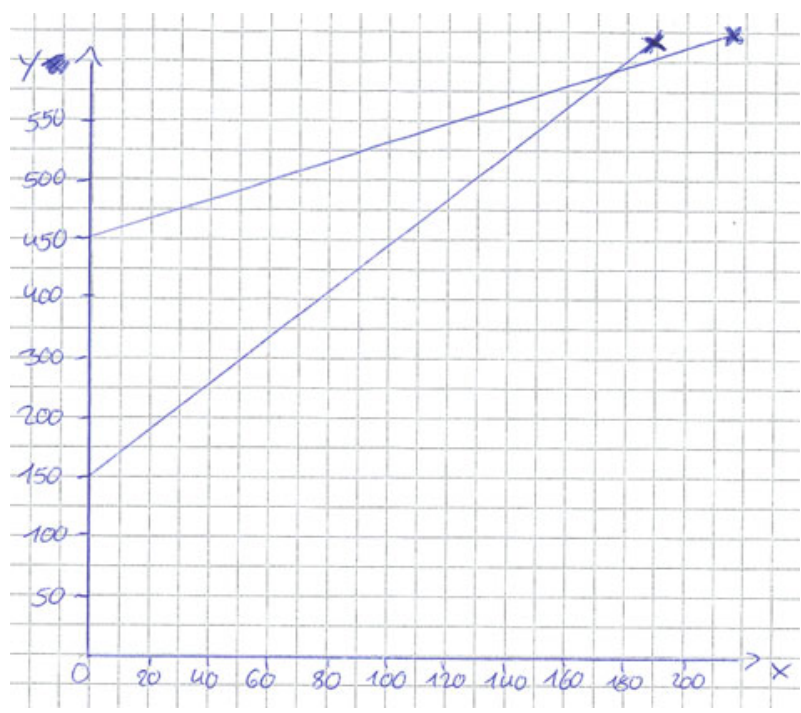




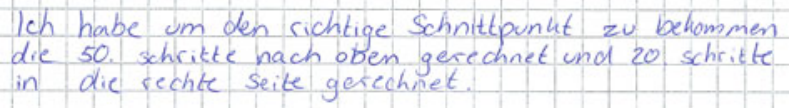

- Abb. 3.3 Schriftliche Konstruktionsbeschreibung (Aufgabe 1b)

Abschrift: „Ich habe um den richtige Schnittpunkt zu bekommen die 50. schritte nach oben gerechnet und 20. schritte in die rechte Seite gerechnet."

Diese Beschreibung verdeutlicht, dass das Steigungsdreieck verwendet wird, um beide Funktionen graphisch darzustellen. Aus inhaltlicher Perspektive ist diese Beschreibung fachlich passend, da formal gesehen die methodische Herangehensweise nachvollziehbar wird. Bei der Betrachtung der grammatischen Zusammensetzung des Satzes wird deutlich, dass die Schülerin Schwierigkeiten sowohl bei der Kommasetzung als auch bei der Nutzung der Zahladverbien sowie bei der Groß- und Kleinschreibung hat. Zudem lässt sich anhand der schriftlichen Erläuterung aufzeigen, dass die Schülerin die Aufgabenstellung, den Schnittpunkt rechnerisch zu bestimmen, fehlinterpretiert hat; das Schriftprodukt hat somit einen kleinen Einblick in den mathematischen Lösungsprozess der Schülerin ermöglicht (vgl. Sjuts 2007).

Analog hierzu können ebenfalls Grenzen beim Formulieren auf Türkisch aufgezeigt werden. Ein Schüler schrieb den in - Abb. 3.4 abgebildeten Antwortsatz.

Es fällt auf, dass der Schüler diesen Satz mit deutschen Buchstaben bildet. Beide Alphabete zeichnen sich zwar durch das lateinische Schriftsystem aus, unterscheiden sich jedoch dahingehend, dass das türkische Alphabet um einige Buchstaben ergänzt wurde. Die Orientierung an deutschen Buchstaben zeigt sich insbesondere dadurch, dass im türkischen Alphabet vorhandene Buchstaben wie das ,1“ nicht genutzt (z. B. sinifina statt sinıfına) und unter den Buchstaben „,c“ uns ,s“, sofern notwendig, keine Cedille gesetzt wurde (z. B. cünkü statt çünkü, tisört statt tişört). Des Weiteren wird der Satzanfang (,ben ...") klein und das Adjektiv „Ilk“ (in: ilk ödeme = die erste Zahlung) großgeschrieben; dies kann als sprachenübergreifender Fehler aufgefasst werden, da sowohl im Türkischen als auch im Deutschen die Satzanfänge groß und die Zahladjektive klein geschrieben werden. Im Rahmen dieser Aufgabenstellung sollte der Schüler Stellung dazu beziehen, welches Angebot die kostengünstigere Variante darstellt. Der Schüler gibt seine Empfehlung ab, bezieht sich dabei jedoch auf keine mathematische Rechnung. Als Begründung werden lediglich Informationen aus der Textaufgabe entnommen und wiedergegeben.

Ein wesentlicher Unterschied zwischen den Bearbeitungen der Mathematikaufgaben in der deutschen und türkischen Sprache besteht im Umfang der Bearbeitungen. Während die erste Aufgabe (auf Deutsch) von allen Schüler*innen in vollem Umfang bearbeitet wurde, ist die zweite Aufgabe (auf Türkisch) von einigen Schüler*innen nur in Ansätzen

ben dsan $10 b$ sinifina 1 tehlifi alirdim cunku bir
tisort fiyati da az ama llu odeme daha coh pagli

- Abb. 3.4 Antwortsatz zu Aufgabe 2d (I)

Abschrift: ,,ben olsam 10b sinifina 1 teklifi alirdim cünkü bir tisört fiyati da az ama Ilk ödeme daha cok pagli“ Übersetzung: „Ich würde das erste Angebot für die Klasse 10b wählen, weil der Einzelpreis eines T-Shirts günstiger ist, aber die erste Zahlung teurer ist“" 


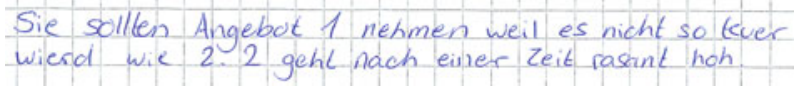

- Abb. 3.5 Antwortsatz zu Aufgabe 1d

Abschrift: „Sie sollten Angebot 1 nehmen weil es nicht so teuer wierd wie 2. 2 geht nach einer Zeit rasant hoh.“

bearbeitet worden. Im Vergleich der auf Deutsch und Türkisch formulierten Antwortsätze fällt auf, dass grundsätzlich die deutschen Antwortsätze Begründungszusammenhänge beinhalten und dadurch im Deutschen längere Sätze formuliert werden. Eine Schülerin notierte in Bezug auf die Frage, welches Angebot der Wohnmobilvermietung die kostengünstigere Variante darstelle (1d), den folgenden Antwortsatz: „Sie sollten Angebot 1 nehmen weil es nicht so teuer wierd wie 2.2 geht nach einer Zeit rasant hoh.“ (- Abb. 3.5).

Es wird deutlich, dass die Schülerin sich eindeutig positioniert und mittels einer Konjunktion ihre Begründung einleitet, indem sie die zuvor erstellten Funktionsgraphen als Argumentationsgrundlage heranzieht.

Bei der Bearbeitung der türkischen Aufgabenstellung formuliert dieselbe Schülerin hinsichtlich der Frage, welches Angebot die Abschlussklasse wählen sollte, den folgenden Antwortsatz: „teklif 1 daha ucuz 2den“ (,Angebot 1 ist günstiger als 2“) (• Abb. 3.6).

Hier wird lediglich eine Aussage getroffen, die auch im weiteren Verlauf nicht begründet wird. Bis auf einen Schüler, der sowohl im Deutschen als auch im Türkischen Begründungszusammenhänge einbringt, ist dieses Phänomen bei den anderen Schüler*innen ebenfalls vorzufinden. Der direkte Vergleich der Antwortsätze zeigt somit auf, dass die Formulierungen der Antwortsätze im Deutschen umfangreicher erfolgten.

Weiterhin konnte beobachtet werden, dass drei der fünf Schüler*innen die zweite Aufgabe auf Türkisch bearbeitet haben. Bei näherer Betrachtung wird jedoch deutlich, dass den Schüler*innen nicht immer die korrekten türkischen Entsprechungen für mathematische Begriffe zur Verfügung standen. Das Subtraktionsverfahren wurde beispielsweise von einem Schüler mit „eksi“ übersetzt, welches jedoch die Bedeutung des Rechenoperators „minus“ hat. Im Vergleich zu den Auffälligkeiten in den deutschen Ausführungen wird deutlich, dass die Herausforderungen der befragten Schüler*innen im Türkischen nicht nur die Grammatik, sondern auch die mathematischen Begrifflichkeiten betreffen. Dies könnte auch eine Erklärung dafür sein, dass nicht alle Schüler*innen die zweite Aufgabe auf Türkisch gelöst haben. Die Ergebnisse verdeutlichen, dass mehrsprachig aufgewachsene Schüler*innen in der herkunftssprachlichen Bildungs- bzw. Fachsprache Defizite aufweisen können, weil bzw. wenn sie diese weder zu Hause noch in der allgemeinbildenden Schule vermittelt bekommen. So gaben zwar alle Schüler*innen an, in der Primarstufe an herkunftssprachlichem Unterricht teilgenommen zu haben; es ist jedoch $\mathrm{zu}$ vermuten, dass dieser nicht fachsprachlich ausgerichtet war bzw. die türkische Bildungssprache nicht in einem ausdifferenzierten Rahmen vermittelt werden konnte. Für

\section{teulif 1 daha vaz 2 den}

- Abb. 3.6 Antwortsatz zu Aufgabe 2d (II)

Abschrift des Antwortsatzes zu Aufgabe 2d: „teklif 1 daha ucuz 2den“

Übersetzung: Angebot 1 ist günstiger als 2 
den Aufbau bildungssprachlicher Kompetenzen in der Herkunftssprache müsste diese in den Lernprozess integriert werden, zum Beispiel durch die Nutzung im Mathematikunterricht oder durch eine Koordination von herkunftssprachlichem Unterricht und Fachunterricht (Meyer und Prediger 2011; Redder et al. 2018; Roll et al. 2019a):

॥ Der Einbezug der Muttersprache in den mathematischen Lernprozess ermöglicht eine Erweiterung der muttersprachlichen (semantischen und lexikalischen) Ressourcen um neu erworbene fachliche Konzepte. Diese Erweiterung ist Voraussetzung zum einen für eine Nutzung des im Mathematikunterricht Gelernten im außermathematischen Denken, zum anderen für eine bildungssprachliche Konsolidierung der Muttersprache in lexikalischer, aber auch struktureller Hinsicht (...) (Meyer und Prediger 2011, S. 187).

Die Ergebnisse aus dem Studienprojekt verdeutlichen, dass sich für die befragten Schüler*innen sowohl auf der fachlichen als auch auf der sprachlichen Ebene Hürden ergeben haben. Die Analyse der fachlichen Verstehensprozesse legt zudem die Vermutung nahe, dass die Schüler*innen unabhängig von der Sprachwahl keine hinreichenden Vorstellungen zu linearen Funktionen aufgebaut haben. Die größten Schwierigkeiten scheinen darin zu bestehen, die aufgestellten Funktionsgleichungen in ein Koordinatensystem zu übertragen und somit einen Darstellungswechsel zu vollziehen sowie die Bedeutung des Schnittpunktes zu erfassen. Diese Aspekte deuten darauf hin, dass die Schüler*innen das Anwenden des Lösungsverfahrens als ein regelhaftes Anwenden bestimmter Operationen verstehen, ohne dabei den Zweck ihrer Handlungen nachzuvollziehen. Um ein tragfähiges Verständnis zu den Lösungsverfahren von Gleichungssystemen aufbauen zu können, sollten Darstellungswechsel in den Unterricht eingebaut, explizit versprachlicht und reflektiert werden. Nur so kann den Schüler*innen ein Zugang zu den weiteren sich anschließenden Fachinhalten eröffnet werden. Die Thematisierung der einzelnen Lösungsverfahren kann mithilfe von grafischen Darstellungen veranschaulicht werden, indem beispielsweise die einzelnen Schritte des Additionsverfahrens in unterschiedliche Koordinatensysteme eingezeichnet werden und ihre Bedeutung reflektiert wird (vgl. Filler 2010, S. 35). Dies könnte den Schüler*innen dazu verhelfen, den Zweck ihrer Handlungen und somit die Sinnhaftigkeit des Lösungsverfahrens zu verstehen und zugleich mit den relevanten sprachlichen Mitteln zu verknüpfen.

Die sprachliche Analyse legt darüber hinaus nahe, dass die befragten Schüler*innen zum Teil Probleme beim Verstehen der Aufgaben hatten. Solche „Lesehürden“ (Prediger et al. 2015, S. 100) konnten anhand des fehlenden Aufgabenverständnisses, wodurch Aufgabenteile entweder gar nicht oder fehlerhaft bearbeitet wurden, identifiziert werden. Um genauere Einblicke in die Verstehensprozesse zu bekommen, könnten mit den Schüler*innen diagnostische Interviews geführt werden (vgl. Gürsoy et al. 2013). Darüber hinaus zeigten sich in den Schüler*innenlösungen in beiden Sprachen grammatische Schwierigkeiten sowie insbesondere im Türkischen lexikalische Unsicherheiten.

Im Rahmen des Praxissemesters konnte innerhalb des Studiums erstmalig eine intensive Auseinandersetzung mit eigenständig erhobenen Lernendenprodukten vorgenommen werden. Die Auseinandersetzung mit Bearbeitungen von Textaufgaben in der deutschen und türkischen Sprache bot interessante Einblicke in diverse fachliche sowie sprachliche Auffälligkeiten. Dass fünf von zehn Schüler*innen die türkische Aufgabenstellung nicht bearbeitet haben, lässt sich möglicherweise auf die sprachlichen Anforderungen in der Bildungssprache Türkisch - insbesondere auf die mangelnde Vertrautheit im Umgang mit türkischsprachigen Mathematikaufgaben sowie auf eine 
gewisse Unsicherheit mit der türkischen Schriftsprache (vgl. Meyer und Prediger 2011, S. $190 \mathrm{f}$.) - zurückführen; hier könnten weitere Untersuchungen anknüpfen. Eine kritische Reflexion dieser Studie zeigt an dieser Stelle allerdings auch Grenzen in der Gestaltung der Lernumgebung. Linguistische und interdisziplinäre Studien belegen, dass mehrsprachige Lernende ihre Sprachen nicht in einsprachiger Logik prozessieren (Gogolin 2006; Rehbein 2011). Darüber hinaus zeigen mathematikdidaktische Studien, wie deutsch-türkische Lernende ohne schulsprachliche Erfahrungen in Einzelsprachen in schriftlichen oder mündlichen Antworten handlungsmustergerechte mathematische Denkprozesse artikulieren können, indem sie beispielsweise auf geschickte und funktionale Weise die Sprachen mischen oder (kognitiv) vernetzen (vgl. Maisano 2019; Wagner et al. 2018; Kuzu 2019). Aus mehrsprachigskeitsdidaktischer Perspektive wäre daher die Nutzung aller Sprachen in gemischtsprachlicher Form empfehlenswert gewesen. Besonders beachtlich ist somit im Rahmen dieses Studienprojektes, dass die Hälfte der befragten Schüler*innen die neuen sprachlichen Herausforderungen bewältigen konnten und die türkische Aufgabenstellung bearbeiteten.

Abschließend ist als besonderes Anliegen dieses Beitrags zu erwähnen, dass sprachlich bedingte Schwierigkeiten nicht nur bei mehrsprachig aufwachsenden Schüler*innen vorzufinden sind. Eine „simplifizierende Gleichsetzung von ,mehrsprachig = sprachlich schwach" verbietet [sich]" (Wessel und Prediger 2017, S. 183), da das alleinige Merkmal der Mehrsprachigkeit nicht losgelöst von den ,zur Verfügung stehenden allgemeinen Entwicklungs- und Bildungsmöglichkeiten“ (Dirim und Mecheril 2010, S. 104) betrachtet werden kann. Wenn Schüler*innen mehrsprachig aufwachsen, stellt sich die Frage, inwiefern die Lerngelegenheiten für die zu erlernenden Sprachen qualitativ und quantitativ ausreichend waren, um diese Sprachen innerhalb bestimmter sozialer Kontexte zu gebrauchen (vgl. Dirim und Mecheril 2010, S. $104 \mathrm{f}$.).

Solche Lerngelegenheiten spielen jedoch nicht nur für bilingual oder mehrsprachig aufwachsende Schüler*innen eine wichtige Rolle, sondern auch für einsprachig aufwachsende. Diese Tatsache erfordert notwendigerweise eine Sensibilisierung der (angehenden) Lehrkräfte für sprachlich bedingte Herausforderungen im Mathematikunterricht. Um verschiedene Schüler*innengruppen nicht unbeabsichtigt im Schulsystem zu benachteiligen, sondern eine Chancengerechtigkeit zu ermöglichen, ist es wichtig, den eigenen Unterricht sprachsensibel zu gestalten, wobei ,integriertes Sprach- und Fachlernen statt[finden]“ (Schmölzer-Eibinger et al. 2013, S. 24) sollte.

\subsection{Sprachbildung in der Lehramtsausbildung Informatik}

Fatma Batur und Jan Strobl

\subsubsection{Einleitung}

Sprachbildung im Informatikunterricht stellt in mehrfacher Hinsicht ein neues und vergleichsweise wenig bearbeitetes Feld dar. Einerseits ist die Menge der wissenschaftlichen Artikel - im deutschsprachigen Raum sei hier insbesondere auf die Veröffentlichungen von Diethelm (z. B. Diethelm et al. 2018) verwiesen - und Praxisveröffentlichungen zu diesem Themenkomplex überschaubar. Andererseits unterliegt das Fach selbst, ebenso wie seine Didaktik, einem steten und schnellen Wandel. Treiber dieses Wandels sind die technologische Weiterentwicklung, die Fachinhalte in deutlich kürzerer Zeit verändert 
und erweitert, als dies in anderen Fächern der Fall ist, und die kontinuierliche Zunahme der Bedeutung des Faches Informatik im Zuge der Durchdringung vieler Bereiche der Gesellschaft mit informatischen Aspekten.

So ist absehbar, dass Informatik zunehmend als verpflichtendes Fach eingeführt und nicht nur wie bislang als Wahl- oder Wahlpflichtfach angeboten wird. Mit diesem Schritt wird sich die Zielgruppe des Informatikunterrichts verbreitern und heterogener werden, da fortan alle Schüler*innen an ihm teilnehmen müssen und nicht mehr von einem relativ hohen Anteil an Schüler*innen mit besonderem Interesse und vorhandenen Vorkenntnissen ausgegangen werden kann.

Im Zuge des „digitalen Wandels“ stellt sich zudem die Frage nach der Breite der Aufgaben des Informatikunterrichts. Soll sich dieser vor allem auf die altersangemessene Vermittlung von Aspekten der Fachwissenschaft Informatik konzentrieren oder soll er auch sozialwissenschaftliche, ethische, politische und andere Aspekte der Digitalisierung im Sinne einer „Digital Literacy“ bündeln? Diese Fragestellung ist auch dafür entscheidend, auf welche Vorarbeiten aus anderen Fächern sich die Entwicklung von Ansätzen für einen sprachbildenden Informatikunterricht stützen kann. Welchen Anteil haben Textsorten, die eher denen der Mathematik ähneln, und welchen haben solche, die eher denen aus gesellschaftswissenschaftlichen Fächern gleichen?

॥ Die Teilhabe an politischen, kulturellen und ökonomischen Prozessen innerhalb der Gesellschaft setzt Fähigkeiten im Umgang mit und zur Analyse, Reflexion und Gestaltung von digitalen Artefakten voraus. Erforderlich hierfür ist die Kenntnis der informatischen Grundlagen sowie der medienwissenschaftlichen und erziehungswissenschaftlichen Zugänge und Diskurse (Brinda et al. 2019, S. 2).

Seit Mitte 2018 besteht an der Universität Duisburg-Essen eine Kooperation zwischen dem Lehrstuhl für Didaktik der Informatik und dem Projekt „ProDaZ. Deutsch als Zweitsprache in allen Fächern“, in der es um diese und weitere Fragestellungen geht.

Die gemeinsame Arbeit umfasst unter anderem Veröffentlichungen, Konferenzbeiträge, beratende Tätigkeiten in Arbeitsgemeinschaften der Bezirksregierungen sowie die universitäre Lehre. Auf die Konzeption eines gemeinsamen Seminars sowie die Erfahrungen einer ersten Durchführung wird im Folgenden eingegangen. Hierfür werden auch einige sprachliche Besonderheiten des Informatikunterrichts thematisiert, um auch einen inhaltlichen Einblick in das Seminar zu geben.

\subsubsection{Seminarkonzeption}

Seit dem Wintersemester 2019/20 wird die Lehrveranstaltung „Sprachbildung im Informatikunterricht" am Lehrstuhl für Didaktik der Informatik als Wahlpflichtfach im Masterstudium angeboten. Da die Anzahl der Studierenden am Lehrstuhl für Didaktik der Informatik im Vergleich zu anderen Fächern meist noch gering ist, sind die Gruppengrößen häufig klein bis sehr klein. Dadurch ergeben sich mehr Freiheiten bei der Seminargestaltung und es ermöglicht eine individuelle Betreuung der Studierenden, jedoch sind kooperative Lernformen, die eine Mindestgruppengröße voraussetzen, nicht oder nur modifiziert durchführbar.

Das interdisziplinäre Seminar „Sprachbildung im Informatikunterricht“ wurde an die Konzeption der Veranstaltung „Konstruktion von Lernumgebungen“ im Fach Mathema- 

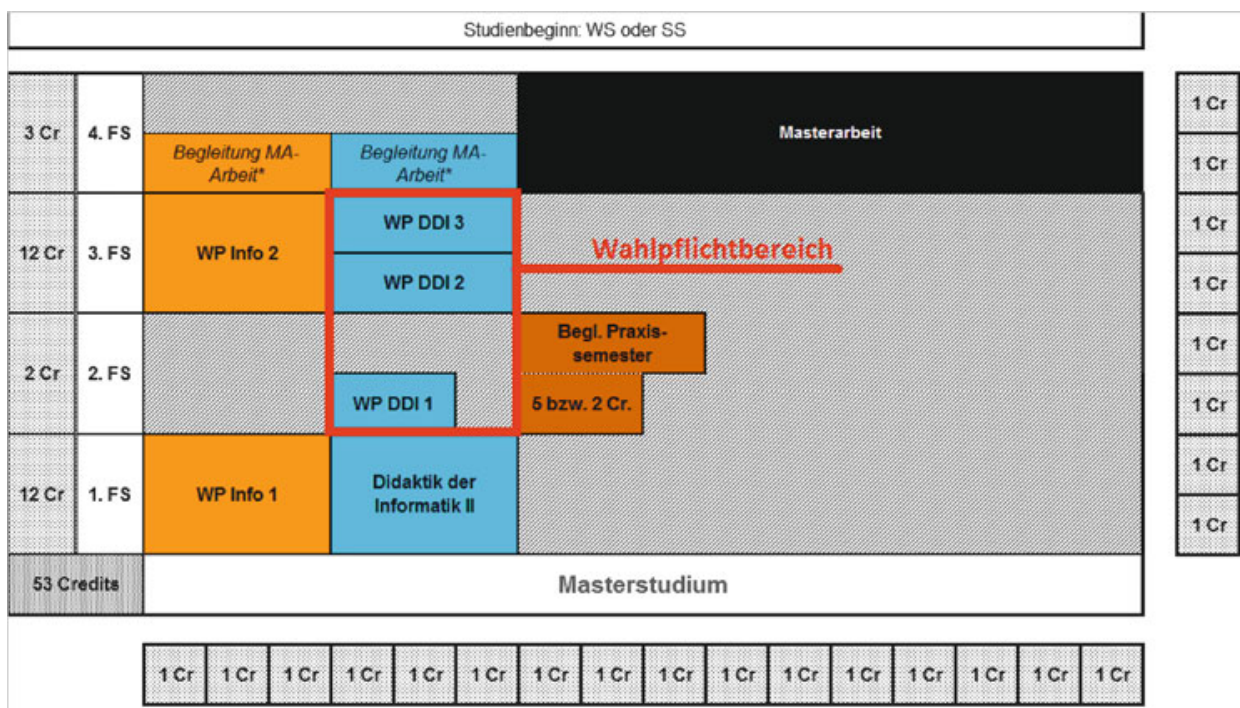

- Begleitveranstaltung im Fach der Master-Arbeit

- Abb. 3.7 Curriculare Verortung des Seminars „Sprachbildung im Informatikunterricht“

tik (vgl. Abschn. 1.3) angelehnt. Das Seminar erweitert das Angebot der DidaktikLehrveranstaltungen im Master-Studiengang Lehramt Informatik (GyGe), um den Studierenden die Möglichkeit zu geben, ihre Kenntnisse aus dem DaZ-Modul ${ }^{1}$ im BachelorStudium fachspezifisch zu vertiefen und mit fachdidaktischen Ansätzen zu verknüpfen.

\section{- Curriculare Verortung}

Master-Studierende des Lehramts Informatik (GyGe) sind verpflichtet, im Bereich Didaktik der Informatik (DDI) insgesamt 14 Creditpoints $(\mathrm{Cr}) \mathrm{zu}$ erwerben, wobei $8 \mathrm{Cr}$ auf den Wahlpflichtbereich entfallen. Dies erreichen sie, indem sie das Modul ,Didaktik der Informatik 2" und drei Seminare aus dem Wahlpflichtbereich (WP DDI 1, WP DDI 2, WP DDI 3; vgl. - Abb. 3.7²) belegen. Durch das Erstere vertiefen sie ihr Wissen zur Fachdidaktik aus dem Bachelor-Studium; die Seminare bieten die Möglichkeit, sich gezielt theorie-, praxis- und unterrichtsorientiert zu spezialisieren. Das Seminar ,Sprachbildung im Informatikunterricht“ ist dabei dem Modul „Unterrichtsorientierte Vertiefung“ zugeordnet. Als Prüfungsleistung erstellen die Studierenden ein semesterbegleitendes Portfolio und stellen einen selbst gewählten Schwerpunkt in einem 30-minütigen Seminarvortrag vor. Das Portfolio enthält dabei u. a. die Ausarbeitung eines selbst gewählten Schwerpunktes (z. B. einen sprachsensiblen Unterrichtsentwurf), die im Laufe des Semesters entstandenen Arbeitsergebnisse (z. B. Analyse von Schulbuchtexten; Aufgabenentwürfe) sowie die Dokumentation und Reflexion des eigenen Lernprozesses.

\section{- Seminargestaltung}

Schwerpunkt der Veranstaltung ist die Thematisierung von Sprachbildung im Fachunterricht Informatik. Am Beispiel verschiedener Themen (wie zum Beispiel objektorientierte Modellierung und Programmierung) werden unterschiedliche Aspekte informati- 
scher Lernumgebungen in sprachbewusster Perspektive thematisiert. Die Studierenden beschäftigen sich mit grundlegenden Methoden und Konzepten des sprachlichen Lernens und machen dieses Wissen für das fachliche Lernen nutzbar. Hierbei werden unterschiedliche Materialien, Aufgaben und Texte auf ihre sprachlichen Anforderungen hin untersucht und herausgearbeitet, wie diese mit Fachkonzepten und fachlichen Anforderungen zusammenhängen und interagieren. Die Studierenden entwickeln anschließend auf Basis der erlernten sprach- und fachdidaktischen Konzepte eigene Ansätze, um das fachliche und sprachliche Lernen $\mathrm{zu}$ verbinden und die sprachlichen Voraussetzungen zum expliziten Lerngegenstand zu machen. Sie entwickeln dabei eigene kleine Unterrichtssequenzen, um bereits erste praktische Erfahrungen mit der Erstellung sprachsensibler Unterrichtsmaterialien zu sammeln. Dazu gehört sowohl die Entwicklung von Aufgaben zur Vertiefung prozessbezogener Kompetenzen (z. B. Modellieren, Analysieren oder Beschreiben), die meist die Anwendung bestimmter sprachlicher Handlungen erfordern, als auch die Gestaltung unterschiedlicher Phasen im Informatikunterricht (Einstiege, Systematisierung und Übung) unter besonderer Berücksichtigung der Sprachbildung.

Die Lehrinhalte des Seminars können folgendermaßen zusammengefasst werden:

- Einführung in die Grundlagen der Sprachbildung im Fach Informatik, u. a. Erarbeitung von Leitlinien für einen sprachaufmerksamen Fachunterricht (vgl. SchmölzerEibinger et al. 2013, S. 20),

- Analyse der gesprochenen und geschriebenen Sprache im Informatikunterricht,

- Sach- und zielgruppengerechte didaktische Aufbereitung von informatischen Inhalten unter besonderer Berücksichtigung von Sprache.

Das didaktische Konzept des Seminars „Sprachbildung im Informatikunterricht“ sieht folgenden Ablauf vor:

- Einführung/Sensibilisierung: Was macht Fachtexte und Sprachverständnis so schwierig?

- Analyse von Schulbuchtexten und Abituraufgaben mit Blick auf sprachliche Herausforderungen

- Analyse der gesprochenen Sprache im Informatikunterricht durch Beobachtung der eigenen Sprache beim Spielen eines Informatik-Spiels (z. B. Informatik-Tabu) unter Einsatz von Videografie bei kleiner Gruppengröße

- Analyse der Operatoren und der dazugehörigen Textsorten und sprachlichen Handlungen im Informatikunterricht der gymnasialen Oberstufe

- Konzepte zur Textproduktion im Informatikunterricht (z. B. Genredidaktik) und Einbindung in den Unterricht

- Sprachstandsdiagnose: Vergleich verschiedener Modelle

- Leseverständnis: Methoden und Strategien zum Lesen und Verstehen von Informatiktexten und -aufgaben

\subsubsection{Ausgewählte Aspekte der Fachsprache im Informatikunterricht}

Beispielhaft werden im Folgenden sprachliche Anforderungen des Informatikunterrichts vorgestellt, mit denen sich die Studierenden im Seminar befassten. Als Einstiegsaufgabe 
zu Beginn des Seminars wurden die Studierenden damit konfrontiert, Darstellungs- und Aufgabentexte ${ }^{3}$ des Informatikunterrichts zu analysieren. Diese Tätigkeit wurde im Laufe des Semesters wieder aufgegriffen. Dabei konnten die Studierenden das Erlernte in ihren Analysen anwenden und diese mit ihrer Lösung der Einstiegsaufgabe abgleichen.

In den Auseinandersetzungen mit Schulbuchtexten und Abituraufgaben stellten die Studierenden fest, dass insbesondere das zielgerichtete Lesen von Texten mit hoher Informationsdichte eine besondere Herausforderung an Schüler*innen im Informatikunterricht stellt. In der Informatik ist die Übertragung solcher Texte in strukturierte Darstellungsformen eine typische Aufgabenstellung. Die Struktur dieser Darstellungsformen unterstützt einerseits beim gezielten Lesen, ist aufgrund des Anspruches an formale und inhaltliche Korrektheit und Vollständigkeit aber auch besonders herausfordernd. Hieran werden einerseits sprachliche Anforderungen deutlich, die sich aus Konzepten des Faches ergeben, andererseits bietet das Fach Werkzeuge an, mit diesen Herausforderungen umzugehen.

Konkret könnten die Schüler*innen im Informatikunterricht mit folgendem Text innerhalb einer Aufgabenstellung konfrontiert sein, wobei sie hieraus z. B. ein Klassendiagramm entwickeln sollen:

॥ Eine Comicfigur besitzt einen Namen und ihr wird als Geburtsjahr das Jahr ihres ersten Erscheinens zugerechnet. Jede Comicfigur ist entweder männlich oder weiblich und hat zudem einen Heimatort, in dem sie lebt. Jede Comicfigur kommt hauptsächlich in einem Comic mit einem Namen als Titel vor. Die Comicfiguren Superhelden haben eine übernatürliche Fähigkeit und einige von ihnen können fliegen, sie alle können aber kämpfen. Die Comicfiguren Helden kämpfen wie Superhelden gegen das Böse und benutzen ein besonderes Hilfsmittel. Die Comicfiguren Tierfiguren sind klar einer Tiergattung zuzuordnen. Die Comicfigur Bösewicht ist der Gegner eines Superhelden oder eines Helden und kann gegen diese kämpfen. Bösewichte können Superbösewichte (ähnlich zu Superhelden) oder normale Bösewichte (ähnlich zu Helden) sein (Kempe et al. 2016, S. 127).

Anhand folgender analytischer Leitfragen, die auch als Fragestellung an die Studierenden formuliert werden können, sollen die genannten Aspekte anhand dieses authentischen Beispiels verdeutlicht werden:

- An welchen Stellen des Textes finden sich die relevanten Elemente des Klassendiagramms und wie sind diese sprachlich codiert?

- Vor welchen Herausforderungen stehen die Lernenden bei deren Identifizierung?

\section{- Welche Informationen müssen im Text identifiziert werden?}

Um ein Klassendiagramm erstellen zu können, müssen Klassen mit ihrer (Vererbungs-) Beziehung sowie ihren Attributen und Methoden gefunden werden. Für die Attribute muss ein geeigneter Datentyp festgelegt werden. Dies setzt zusätzlich zum Textverständnis ein entsprechendes Kontextwissen voraus, um zu erkennen, um welche Art von Wert (z. B. Zahl, Datum oder Text) es sich handelt. Häufig müssen auch Eingabe- und Rückgabewerte von Methoden gefunden werden, hiervon wird in diesem Beispiel aber abgesehen.

3 Kapitel aus verschiedenen Informatik-Schulbüchern für die Sekundarstufen I und II. 
Exkurs: Objektorientierung und ihre Darstellungsformen

Das in den Leitfragen genannte Klassendiagramm ist ein Modell der objektorientierten Programmierung. Die objektorientierte Programmierung (OOP) (vgl. Kay 1993) ist ein Programmierparadigma, bei dem der zu programmierende Sachverhalt mit sogenannten Objekten abgebildet wird und das für viele moderne Programmiersprachen - auch die im Schulunterricht am häufigsten verwendete Sprache Java - zentral ist.

Auch innerhalb der Objektorientierung existieren - wie oben dargestellt - verschiedene Darstellungsformen, die nach Anwendungsfall ineinander überführt werden müssen (vgl. - Abb. 3.8).

Im Kontext der Objektorientierung sind Objekte dabei stets konkrete Instanzen bestimmter Klassen. So ist z. B. eine Klasse Fahrzeug denkbar. Klassen wiederum können bestimmte Merkmale von Objekten - die Attribute - vorgeben, im Beispiel Bauj ahr oder Gewicht. Ebenso können bestimmte Aktivitäten von Objekten vorgesehen werden: hierfür werden Methoden definiert. Mehrere Klassen mit gemeinsamen Eigenschaften können von einer gemeinsamen Superklasse/Oberklasse abgeleitet sein, so können z. B. Fahrrad und Auto Spezialisierungen einer Klasse Fahrzeug sein, dies wird gemeinhin als Vererbung bezeichnet. Die Subklassen/Unterklassen weisen dabei alle Eigenschaften (Methoden und Attribute) der allgemeineren Klasse auf, erweitern diese aber um zusätzliche.

Ein Sachverhalt aus der realen Welt (• Abb. 3.8, links) wird hierbei - häufig - zunächst abstrahiert in einem Modell dargestellt - das bezeichnet man als informatisches Modellieren. Das Ergebnis des Modellierens ist in diesem Beispiel ein Klassendiagramm (- Abb. 3.8, Mitte oben), in dem die Klassen mit ihren Attributen, Methoden und Vererbungsbeziehungen in einer bestimmten Modellsprache (vgl. OMG 2017) dargestellt werden.

Ein solches Modell kann u. a. beschrieben ${ }^{4}$, analysiert oder interpretiert werden, wofür entsprechende sprachliche Handlungen und Textsorten beherrscht und folglich zuvor erlernt werden müssen. Wird der Sachverhalt - entweder direkt oder auf Basis des Modells - in eine Programmiersprache (vgl. - Abb. 3.8, rechts) übersetzt, so wird dies als Implementieren bezeichnet (vgl. QUA-LiS NRW 2018).

\section{- Klassen im Text erkennen}

Basis eines Klassendiagramms sind stets die Klassen. Es wird üblicherweise davon ausgegangen, dass diese als Substantive (in - Abb. 3.9 roter Text) im Text zu finden sind ${ }^{5}$.

Tatsächlich sind alle Klassen (in - Abb. 3.9 gelb hinterlegt) als Substantive im Text zu finden, jedoch finden sich auch weitere Substantive im Text.

\section{- Attribute im Text erkennen}

Hierbei kann es sich um Attribute (in - Abb. 3.9 hellgrau hinterlegt) handeln, es könnten jedoch auch andere nicht-modellrelevante Substantive sein. Da es sich bei den Attributen um Eigenschaften aller durch die Klasse spezifizierten Objekte handelt, sind hier Formulierungen mit haben, besitzen usw. zu erwarten. Attribute können jedoch auch als

4 Das Beschreiben eines Klassendiagramms, also die Übersetzung von einem Klassendiagramm (• Abb. 3.8, Mitte oben) in eine Diagrammbeschreibung (- Abb. 3.8, Mitte unten) unter Verwendung eines genredidaktischen Ansatzes, wurde bereits an anderer Stelle publiziert (vgl. Batur und Strobl 2019).

5 Die „Abbott Textual Analysis“ (Abbott 1983) ist ein häufig verwendetes Verfahren in der Informatik. 

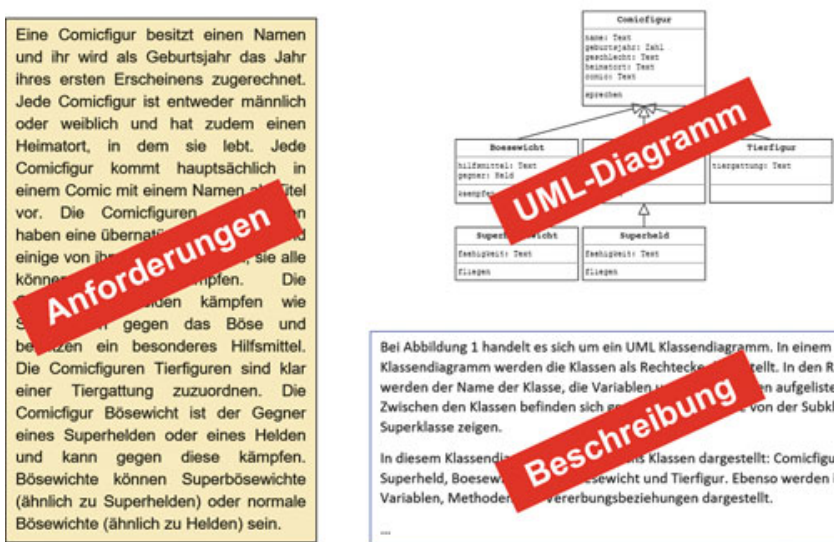

Bei Abbildung 1 handelt es sich um ein UML Klassendiagramm. In einem UMLKlassendiagramm werden die Klassen als Rechteckor tellt. In den Rechtecken werden der Name der Klasse, die Variablen no naufgelistet. zwischen den Klassen befinden sich p. WuIn der Subklasse zur Superklasse zeigen.

In diesem Klassendin $\quad 8 \mathrm{O}^{5}$ is Klassen dargestellt: Comicfigur, Held, Superheld, Boesew BO Variablen, Methoder vererbungsbeziehungen dargestellt.

- Abb. 3.8 Unterschiedliche Darstellungsformen in der Objektorientierung (eigene Darstellung). (Im ProDaZJournal 3 (vgl. Batur und Strobl 2020) findet sich ein weiteres Beispiel für unterschiedliche Darstellungsformen im Informatikunterricht)

Eine Comicfigur besitzt einen Namen und ihr wird als Geburtsjahr das Jahr ihres ersten Erscheinens zugerechnet. Jede Comicfigur ist entweder männlich oder weiblich und hat zudem einen Heimatort, in dem sie lebt. Jede Comicfigur kommt hauptsächlich in einem Comic mit einem Namen als Titel vor. Die Comicfiguren Superhelden haben eine übernatürliche Fähigkeit und einige von ihnen können fliegen, sie alle können aber kämpfen. Die Comicfiguren Helden kämpfen wie Superhelden gegen das Böse und benutzen ein besonderes Hilfsmittel. Die Comicfiguren Tierfiguren sind klar einer Tiergattung zuzuordnen. Die Comicfigur Bösewicht ist der Gegner eines Superhelden oder eines Helden und kann gegen diese kämpfen. Bösewichte können Superbösewichte (ähnlich zu Superhelden) oder normale Bösewichte (ähnlich zu Helden) sein.

- Abb. 3.9 Aufgabentext mit Markierungen (Kempe et al. 2016)

mögliche Merkmalsausprägungen - wobei der Name des Attributs dann selbst festgelegt werden muss - also meist als Adjektive (hier männlich oder weiblich) im Text zu finden sein. Das Erkennen von Attributen ist äußerst komplex. Die folgende - Tab. 3.1 listet die Vielzahl an Formulierungen auf, anhand derer in diesem Text Attribute erkannt werden müssen.

Besonders herausfordernd sind in diesem Text die weiteren inhaltlichen Erläuterungen wie ,ihr wird als Geburtsjahr das Jahr ihres ersten Erscheinens zugerechnet“ und „Comicfigur kommt hauptsächlich in einem Comic mit einem Namen als Titel vor", die selbst für geübte Lesende kaum verständlich sind. Vor allem ,zurechnen“ kann in dem Fall leicht missverstanden werden, da hier zwei Zahlenwerte einander zugeordnet werden, die jedoch, gerade im Kontext der Fächer Informatik und Mathematik, auch miteinander addiert ${ }^{6}$ werden könnten.

6 Der Duden nennt drei mögliche Bedeutungen von „zurechnen“: die hier gemeinte Zuordnung, die hier leicht anzunehmende Addition und ,zur Last legen“: $\gg$ https://www.duden.de/rechtschreibung/zurechnen. 
- Tab. 3.1 Formulierungen, die Attribute anzeigen können

\begin{tabular}{|c|c|c|}
\hline Formulierung & Bemerkung & Beispiel \\
\hline ... besitzt einen $\ldots$ & & Namen \\
\hline $\begin{array}{l}\text { Ihr wird als } \ldots \\
\text { rechnet }\end{array}$ & $\begin{array}{l}\text { Wird um Angabe ergänzt, welcher } \\
\text { Wert (hier dunkelgrau hinterlegt) } \\
\text { dem Attribut zugewiesen wird. }\end{array}$ & $\begin{array}{l}\text { Geburtsjahr (Attribut) } \\
\text { das Jahr ihres ersten } \\
\text { Erscheinens (Wert) }\end{array}$ \\
\hline$\ldots$ ist entweder $\ldots$ oder & $\begin{array}{l}\text { Es ist keine Angabe des Attributs, } \\
\text { sondern nur die Angabe zweier Aus- } \\
\text { prägungen vorhanden. (Könnte damit } \\
\text { auch als Boolean modelliert werden.) }\end{array}$ & $\begin{array}{l}\text { Geschlecht (Attribut, muss } \\
\text { selbst erschlossen werden) } \\
\text { männlich/weiblich (Wert) }\end{array}$ \\
\hline $\begin{array}{l}\text { Jede } \ldots \text { hat eine } \ldots \text { / hat } \\
\text { einen } \ldots\end{array}$ & & Heimatort \\
\hline $\begin{array}{l}\ldots \text { haben eine } \ldots \text { / haben } \\
\text { einen } \ldots\end{array}$ & Name der Klasse im Plural & Fähigkeit \\
\hline $\begin{array}{l}\text { Kommt hauptsächlich in einem } \\
\ldots \text { vor }\end{array}$ & & Comictitel \\
\hline Benutzen ein $\ldots$ & & Hilfsmittel \\
\hline $\begin{array}{l}\text { Sind einer/einem } \ldots \text { zuzuord- } \\
\text { nen }\end{array}$ & $\begin{array}{l}\text { Die Formulierung könnte leicht als } \\
\text { Vererbung gelesen werden. }\end{array}$ & Tiergattung \\
\hline $\begin{array}{l}\ldots \text { ist der/die/das } \ldots \\
\text { eines/einer } \ldots\end{array}$ & $\begin{array}{l}\text { Der Attributwert ist eine Referenz auf } \\
\text { ein Objekt einer anderen (oder ggfs. } \\
\text { der eigenen) Klasse. }\end{array}$ & Gegner \\
\hline
\end{tabular}

\section{- Methoden im Text erkennen}

Methoden (in - Abb. 3.9 cyan hinterlegt), also die durch die Klasse vorgegebenen möglichen Aktionen bzw. Fähigkeiten von Objekten, sind häufig als Verben im Text zu finden. In diesem Text sind sie recht leicht zu erkennen, da sie überwiegend durch das Modalverb „können“ markiert werden.

\section{- Vererbungsbeziehungen im Text erkennen}

Vererbungsbeziehungen zwischen Klassen (in - Abb. 3.9 unterstrichen), also Spezialisierungen und Verallgemeinerungen von Klassen (z. B. Superheld, Held, Comicfigur), müssen im Text ebenfalls erkannt werden. Hierfür werden im Text enge Appositionen wie „Die Comicfiguren Helden“ verwendet, die anzeigen, dass Helden Comicfiguren sind. Andere typische Formulierungen wären z. B. ,ein Held ist eine Comicfigur“/,Helden sind Comicfiguren“ oder „Comicfiguren können Helden, Bösewichte oder Tierfiguren sein“.

Als Ergebnis der Modellierung entsteht ein Klassendiagramm mit Klassen, Attributen, Methoden und Vererbungsbeziehungen (• Abb. 3.10).

Eine weitere Schwierigkeit taucht bei der Benennung der Klassen auf. Während im (deutschsprachigen) Schulunterricht auch von Ober- und Unterklassen (vgl. QUA-LiS NRW 2018) gesprochen wird, ist in der Fachliteratur (vgl. Lemay und Cadenhead 2005, S. 47) und im Internet häufig von Sub- und Superklassen (bzw. auf Englisch sub- und 


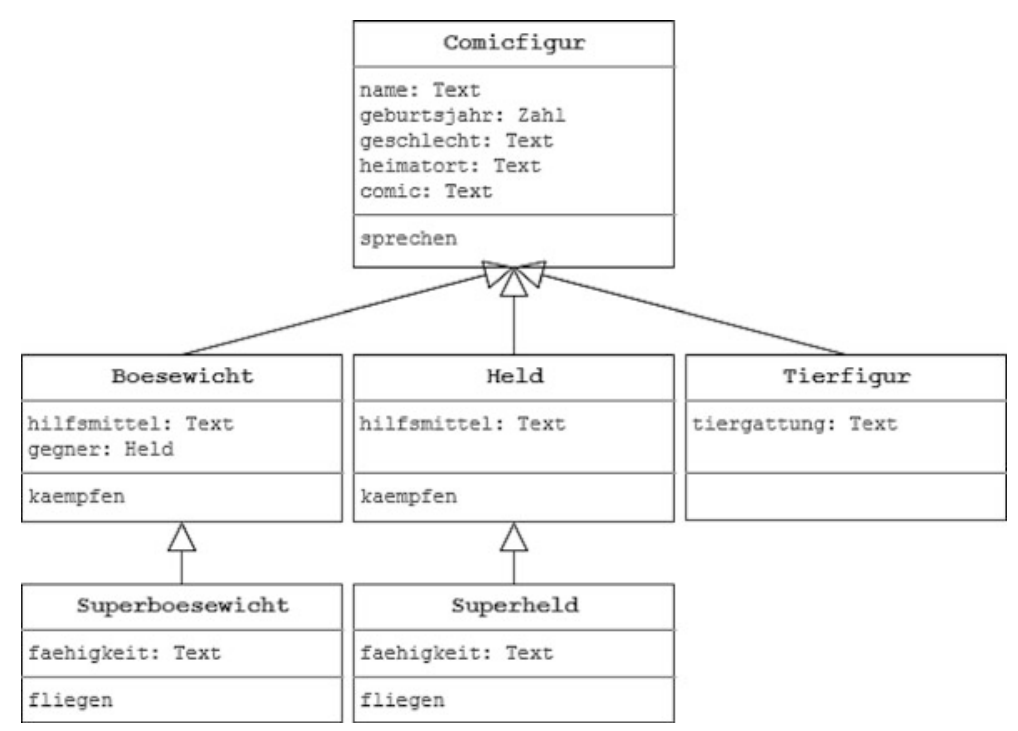

- Abb. 3.10 Klassendiagramm „Comicfigur“ (eigene Darstellung)

superclass) die Rede. Eine Ober- oder Superklasse ist dabei die nächsthöhere Klasse innerhalb der Vererbungshierarchie (vgl. - Abb. 3.11), z. B. ist Held die Superklasse von Superheld und Superheld eine Subklasse von Held.

Hier könnte es zu Verwirrung kommen, da Superheld und Superboesewicht in keinem Zusammenhang Superklassen sind (vgl. - Abb. 3.11). Hier zeigt sich ein - auch sprachlicher - Lerninhalt der Informatik, nämlich die Rolle sogenannter Bezeichner (z. B. Superheld). Bezeichner sind so etwas wie Eigennamen innerhalb von Programmen, die zunächst einmal beliebig gewählt werden können und keinen Einfluss auf Inhalt und

- Abb. 3.11 Widerspruch zwischen Bezeichner und tatsächlicher Vererbungsbeziehung (eigene Darstellung)

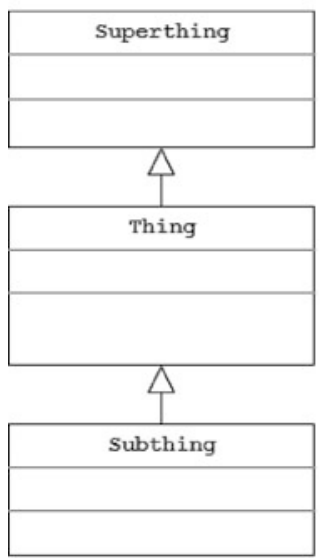


Eigenschaften des von ihnen bezeichneten Gegenstandes haben. Wie etwas heißt und was es ist, sollte aus Lesbarkeitsgründen in den meisten Fällen zwar schon etwas miteinander zu tun haben, es muss technisch aber nicht der Fall sein. Im Extremfall kann, wie in diesem Beispiel, bei bestimmter Lesart das Gegenteil der tatsächlichen Eigenschaft durch den Bezeichner suggeriert werden.

\subsubsection{Erste Erfahrungen und Ausblick}

Nach dem ersten Durchlauf bekundeten die Seminarteilnehmer*innen in der abschließenden Gruppendiskussion, dass sich ihr Blick auf die Fachtexte des Informatikunterrichts geschärft hätte und somit ein bewussterer Umgang damit angeregt wurde. Insbesondere von der Textlastigkeit der Abituraufgaben im Fach Informatik zeigten sich die Studierenden überrascht und merkten an, dass dies die Lernenden vor große Herausforderungen stellt, auf die sie explizit vorbereitet werden sollten. Auch mit den von Aufgabe zu Aufgabe stark unterschiedlichen Anforderungen und teils verwirrenden, eigentlich der Veranschaulichung dienenden Real-Welt-Bezügen setzten sie sich kritisch auseinander. Zur Vertiefung entwickelten die Studierenden unter anderem Materialien zur Unterstützung der Lesekompetenz in einer Unterrichtsstunde zum Thema „Algorithmen“ oder einen genredidaktischen Ansatz für das Schreiben natürlichsprachlicher Erläuterungen von „Methoden in Programmen“.

Die Kooperation zwischen dem Lehrstuhl Didaktik der Informatik und dem Projekt ProDaZ soll in Zukunft weiter ausgebaut werden. Dabei wird das erstmalig im WS 2019/20 angebotene Seminar „Sprachbildung im Informatikunterricht“ weiterentwickelt und in einem jährlichen Turnus verstetigt. Weiterhin werden Abschlussarbeiten (Bachelor- sowie Masterarbeiten) zu dem Themenbereich „Sprachbildung im Informatikunterricht“/,Sprachsensibler Informatikunterricht" vergeben. Die Ergebnisse dieser Arbeiten sollen u.a. an die bereits erfolgten Analysen anknüpfen und weiterführende Vertiefungen ermöglichen.

Die Studierenden werden im Rahmen des Vorbereitungsseminars für das Praxissemester die Möglichkeit erhalten, ihr Studienprojekt an diesem Themenbereich auszurichten. Dies ermöglicht den Studierenden bereits in der ersten längeren Praxisphase ihrer Lehramtsausbildung verschiedene Ansätze und Konzepte aus dem Seminar im Unterricht anzuwenden und diese zum Beispiel durch teilnehmende Beobachtung oder andere Methoden zu evaluieren. Zudem gewinnt die Mündlichkeit im Informatikunterricht durch die Einführung des Pflichtfachs Informatik eine besondere Bedeutung, sodass bei Studienprojekten beispielsweise der Fokus auf die Teilfertigkeiten Sprechen und Hörverstehen gesetzt werden könnte.

Neben weiteren eigenen Veröffentlichungen und Tagungsbeiträgen ist die Entwicklung von Lehrer*innenfortbildungen vorgesehen. Dabei werden die Forschungsergebnisse und aber auch die Arbeitsergebnisse aus der Lehrveranstaltung für die inhaltliche Gestaltung verwendet. 


\subsection{Chemische Konzepte und Sprache im Übergang - Ein Seminarkonzept zur praxisnahen Ausbildung von Lehramtsstudierenden in den Fächern Sachunterricht und Chemie in der Sekundarstufe I}

Melanie Beese, Dennis Kirstein und Henning Krake

\subsubsection{Einleitung}

Im Rahmen dieses Beitrages wird eine gemeinsame Master-Veranstaltung für die Lehramtsstudiengänge Sachunterricht (Grundschule) und Chemie (Haupt-, Real-, Sekundarund Gesamtschule) beschrieben, in der die Studierenden Lernsequenzen mit 60 min Länge für beide Schulformen planen, durchführen und reflektieren. Ein Ziel der Veranstaltung ist, die Möglichkeiten und Notwendigkeiten des sprachsensiblen Unterrichtens chemischer Inhalte aufzuzeigen. Ein besonderer Fokus wird dabei auf den Übergang vom naturwissenschaftlichen Sachunterricht zum chemischen Fachunterricht gelegt. Durch eine schulformübergreifende Gruppenarbeit wird die Heterogenität der Teilnehmenden unter anderem in Bezug auf Studienfach, chemisches Fachwissen und fächervernetzendes Lernen gewinnbringend aufgegriffen. Auch sehen sich die angehenden Lehrkräfte im späteren Beruf einem wachsenden Bedarf an sprachsensiblem Unterricht gegenüber. Die verstärkte Verzahnung von sprachlichem und fachlichem Lernen wird auf Dozierendenseite durch eine Kooperation der Didaktik der Chemie mit dem Institut DaZ/DaF erreicht und spiegelt sich in den geplanten Lernsequenzen wieder. Im Rahmen je eines Entdeckertages, bei dem je eine Grund- und eine Gesamtschulklasse die Universität besucht, haben die Studierenden zudem die Gelegenheit, ihre geplanten Lernsequenzen in der Praxis zu erproben und im Anschluss videogestützt zu reflektieren.

\subsubsection{Planung von Unterricht}

Den zeitlichen wie inhaltlichen Kern der Veranstaltung bildet die theoriebasierte Planung von Unterricht mit chemischen Fachinhalten. Um den Unterricht möglichst lernwirksam zu gestalten, ist es Aufgabe der Studierenden, zentrale Bausteine zu berücksichtigen, die sich für den (experimentgestützten) Unterricht chemischer Inhalte als förderlich erwiesen haben bzw. curricular bindend sind. Dazu zählen die Kontextorientierung, der kumulative Wissensaufbau auf Basis einer Learning Progression, kognitive Aktivierung und inhaltliche Strukturierung als zentrale Qualitätsmerkmale des naturwissenschaftlichen Unterrichts und die Verzahnung von sprachlichem und fachlichem Lernen. Diese Bausteine sind jedoch nur sehr bedingt isoliert zu betrachten, sondern weisen Bezüge zueinander auf und beeinflussen sich immer wieder gegenseitig.

\section{- Kontextorientierung}

Den Ausgangspunkt der Unterrichtsplanungen bildet das chemiedidaktische Konzept Chemie im Kontext (ChiK) (Demuth et al. 2008). Ziel ist, dass ein chemisches Konzept nicht rein fachlich, sondern ausgehend von einem für die Lernenden persönlich oder gesellschaftlich relevanten Kontext behandelt wird, der einen zentralen Anreiz und Be- 
zugspunkt für die Erarbeitung chemischer Fachinhalte darstellt. Van Vorst et al. (2015) haben sowohl Erklärungsansätze zur positiven Wirkung von Kontexten auf das Interesse der Lernenden und zur intendierten Steigerung der Lernleistung wie auch Merkmale von Kontexten zusammengefasst, die helfen, die Eignung eines Kontextes zu bewerten. Zunächst gilt es also für die Studierenden, geeignete Kontexte auf Basis der Merkmale Bekanntheit, Relevanz, Komplexität und Authentizität (van Vorst, et al. 2015) auszuwählen und auch auf ihren fachlichen Hintergrund hin zu beschreiben. Der Bezug zur Lebenswelt, der über die Kontextorientierung hergestellt wird, eröffnet auch einen $\mathrm{Zu}$ gang zur Auseinandersetzung mit alltags- und fachsprachlichen Phänomenen, sodass hier eine Anknüpfung an den Planungsbaustein sprachliches und fachliches Lernen existiert.

Das Konzept Chemie in Kontext basiert neben der Säule „Kontextorientierung“ auf den Säulen „Vernetzung zu Basiskonzepten“ und „Unterrichtsgestaltung“. Diese werden in den folgenden Abschnitten aufgegriffen.

\section{- Learning Progression}

Die Säule „Vernetzung zu Basiskonzepten“ sieht vor, dass das im Rahmen des Kontextes erworbene Wissen dekontextualisiert, das heißt in bestehende Konzepte integriert wird, um es leichter auf andere Kontexte übertagbar zu machen und einen kumulativen Wissensaufbau zu ermöglichen. Der Kernlehrplan für das Fach Naturwissenschaften an Gesamtschulen in Nordrhein-Westfalen (NRW) (Ministerium für Schule und Weiterbildung des Landes Nordrhein-Westfalen 2012) benennt drei Basiskonzepte: Struktur der Materie, Energie und Chemische Reaktion. Für diese drei Basiskonzepte wurde von Celik und Walpuski (2018) eine Learning Progression in Form einer Strand Map entwickelt und evaluiert (Celik und Walpuski 2018; Rother und Walpuski 2020), die den Aufbau der Basiskonzepte im Laufe der Sekundarstufe I in Form von aufeinander aufbauenden Kernideen beschreibt. Diese Learning Progression wurde von dem Autorenteam dieses Artikels um die chemischen Kernideen des Sachunterrichts an Grundschulen in NRW erweitert (siehe - Abb. 3.12).

In der Abbildung sind die drei Basiskonzepte farblich kodiert. Die Verwendung von Mischfarben (lila und orange) soll hier andeuten, dass eine eindeutige Zuordnung ausgewählter Kernideen zu einem Basiskonzept nicht immer funktioniert. Die gestrichelte Linie markiert den Übergang zwischen Sach- und Fachunterricht. Im Gegensatz zur Learning Progression für die Sekundarstufe I ist der Teil für die Grundschule bisher ein Arbeitspapier, dessen wissenschaftliche Evaluation aber in Planung ist.

Bereits jetzt erweist sich die Strand Map als wertvolle Orientierung bei der Planung der Lernsequenzen. Die Arbeit mit der Learning Progression hilft den Studierenden sowohl bei der Lernzielbestimmung als auch dabei, das aktive Einbringen von Vorwissen zu unterstützen, ein zentrales, lernwirksames Qualitätsmerkmal des experimentgestützten Chemieunterrichts (Schulz 2011). Die Strand Map unterstützt die Studierenden auch bei der Gestaltung des Übergangs von der Grundschule zur Sekundarstufe I. Die Studierenden haben die Aufgabe, die Lernsequenzen für die Grundschule und die Sekundarstufe I anhand der aufeinander aufbauenden Kernideen eines Strangs zu planen. Dabei gibt eine Kernidee den lower anchor an, das heißt das Vorwissen der Lernenden, und die darüber liegende Kernidee den upper anchor, also das Lernziel der Lernsequenz (Durschl et al. 2011). Das Lernziel der Lernsequenz der Grundschule ist dann in der Regel der lower anchor für die Lernsequenz der Sekundarstufe I. Mit den jeweiligen Kernideen sind ebenfalls ein charakteristischer Wortschatz, spezifische Sprachmuster sowie teilweise Darstellung in Modellform verbunden. Damit hilft die Strand Map außerdem dabei, 


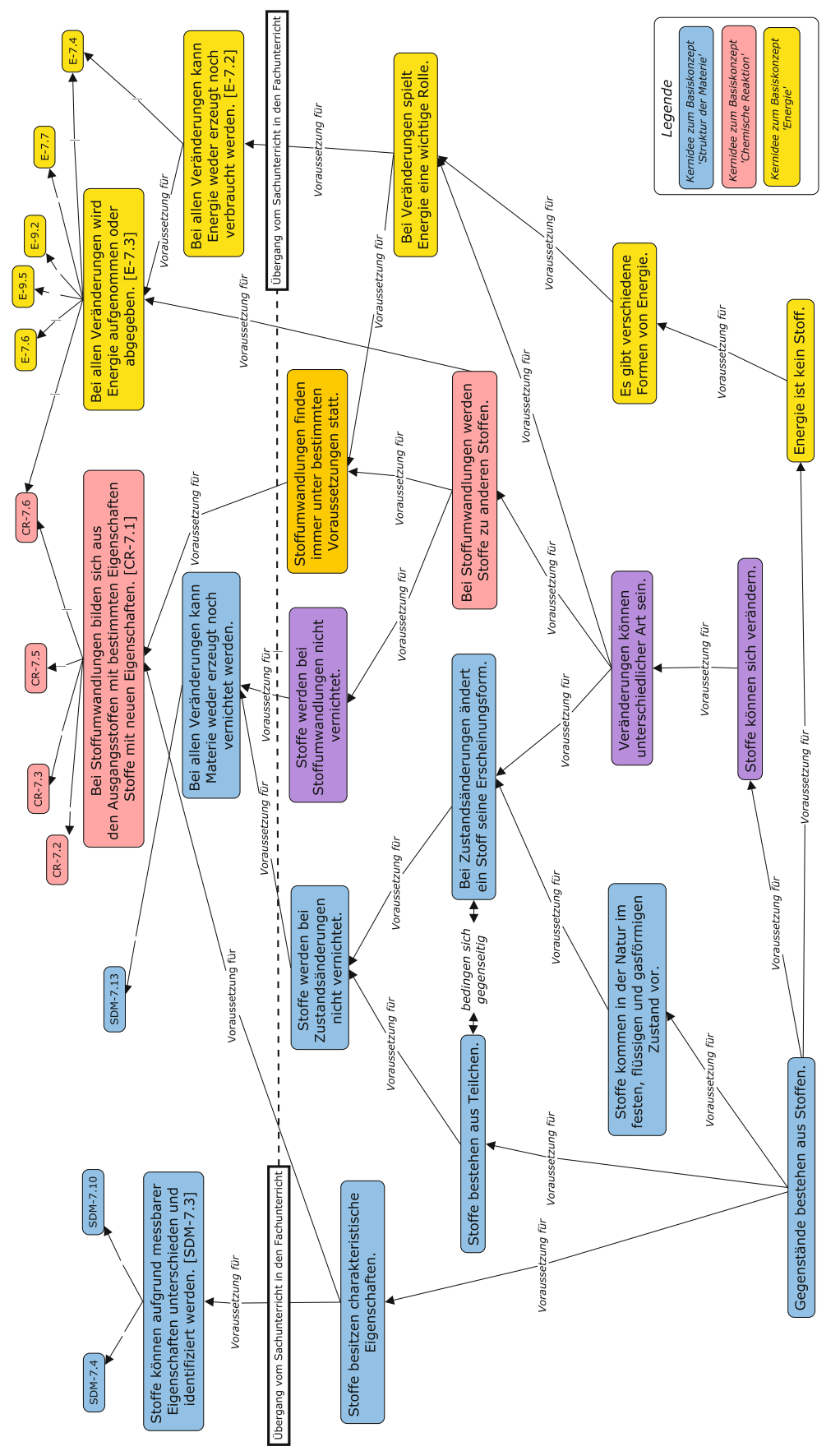

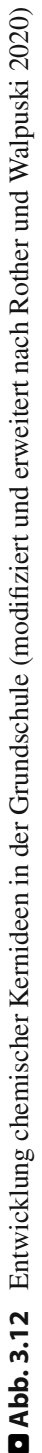


wesentliche sprachliche Lernziele zu identifizieren und den Übergang auf sprachlicher Ebene zu gestalten.

\section{- Kognitive Aktivierung und inhaltliche Strukturierung}

Den Unterricht unter Berücksichtigung lernwirksamer Qualitätsmerkmale für naturwissenschaftlichen Unterricht zu gestalten, ist ein weiterer Baustein der Planung von Unterricht. Als zentrale Qualitätsdimensionen werden hier in Anlehnung an Kleickmann (2012) sowie Klieme et al. (2009) kognitive Aktivierung und inhaltliche Strukturierung aufgegriffen.

Bei der kognitiven Aktivierung werden im Seminar die Punkte problemorientierte Aufgabenstellungen und Lebensweltbezug, aktives Einbringen von Vorwissen und die Kommunikation bzw. Kooperation zwischen Lernenden thematisiert (Kleickmann 2012).

Zur inhaltlichen Strukturierung wird den Studierenden als Planungsunterstützung die Möglichkeiten der Tiefenstrukturierung der Lernsequenzen in Anlehnung an die Basismodelle des Lehrens und Lernens von Oser (Oser und Baeriswyl 2001) vorgestellt. In der physikdidaktischen Forschung wurden für drei der zwölf Basismodelle die besondere Bedeutung und deren Lernwirksamkeit hinreichend belegt (Zander 2016; Maurer 2016; Geller 2015). Diese drei werden im Seminar vorgestellt: Lernen durch Eigenerfahrung, Problemlösen, Begriffs- und Konzeptbildung. Für das Seminar wurden die Basismodelle so angepasst, dass jeweils eine experimentelle Phase vorgesehen ist (siehe $\mathbf{0}$ Tab. 3.2).

- Tab. 3.2 Basismodelle des naturwissenschaftlichen Unterrichts mit Experiment (modifiziert nach Oser und Baeriswyl 2001)

\begin{tabular}{|c|c|c|c|}
\hline \multirow[t]{2}{*}{ HKS } & \multicolumn{3}{|c|}{ (Kognitive) Aktivität des Handlungskettenschrittes (HKS) } \\
\hline & $\begin{array}{l}\text { Basismodell 1: Lernen } \\
\text { durch Eigenerfahrung }\end{array}$ & $\begin{array}{l}\text { Basismodell 3: } \\
\text { Problemlösen }\end{array}$ & $\begin{array}{l}\text { Basismodell 4: Begriffs- } \\
\text { und Konzeptbildung }\end{array}$ \\
\hline 1 & $\begin{array}{l}\text { Planung des Experiments } \\
\text { (guided/open inquiry) }\end{array}$ & $\begin{array}{l}\text { Problemgenerierung durch } \\
\text { Diskrepanzerlebnis (z. B. } \\
\text { unerwartete Beobachtung } \\
\text { bei Experiment) }\end{array}$ & $\begin{array}{l}\text { Rückgriff auf und Bewusst- } \\
\text { machung von Vorwissen }\end{array}$ \\
\hline 2 & $\begin{array}{l}\text { Durchführung des Experi- } \\
\text { ments, Beobachtungen } \\
\text { machen }\end{array}$ & $\begin{array}{l}\text { Problemformulierung durch } \\
\text { Lernende, um Problem- } \\
\text { verständnis zu zeigen } \\
\text { (guided/open inquiry) }\end{array}$ & $\begin{array}{l}\text { Durchführung eines } \\
\text { Experiments }\end{array}$ \\
\hline 3 & $\begin{array}{l}\text { Auswertung des Experiments, } \\
\text { Deuten der Beobachtungen }\end{array}$ & $\begin{array}{l}\text { Planen von Experimenten zur } \\
\text { Lösung des Problems }\end{array}$ & $\begin{array}{l}\text { Sammeln der Beobachtungen } \\
\text { und Auswerten des Experi- } \\
\text { ments (Explizierung des } \\
\text { neuen Begriffs/Konzepts) }\end{array}$ \\
\hline 4 & $\begin{array}{l}\text { Deduktion/Verallgemeinerung } \\
\text { der exemplarischen Auswer- } \\
\text { tung }\end{array}$ & $\begin{array}{l}\text { Durchführung der Experi- } \\
\text { mente }\end{array}$ & $\begin{array}{l}\text { Einordnung und In-Bezie- } \\
\text { hung-Setzen des neuen } \\
\text { Begriffs/Konzepts zu } \\
\text { bereits Bekanntem }\end{array}$ \\
\hline 5 & $\begin{array}{l}\text { Herstellen eines Alltags- } \\
\text { bezugs/Einbettung in ein } \\
\text { Konzept bzw. einen Kontext }\end{array}$ & $\begin{array}{l}\text { Evaluation des Lösungsweges } \\
\text { - Abstraktion/Verallgemei- } \\
\text { nerung/Anwendung des } \\
\text { Lösungsweges }\end{array}$ & $\begin{array}{l}\text { Transfer auf/Verwendung des } \\
\text { neuen Begriffs in anderen } \\
\text { Kontexten }\end{array}$ \\
\hline
\end{tabular}


Die Basismodelle bieten den Studierenden auf der einen Seite Unterstützung, da die lernzielabhängigen Prozessschritte (Handlungskettenschritte) vorgegeben sind, die methodische Ausgestaltung aber für die Studierenden offen bleibt und die Möglichkeit der Anpassung an die Lernenden bietet. Das Basismodell 4 „Begriffs- und Konzeptbildung“ wird dabei von den Studierenden mit Abstand am häufigsten gewählt.

\section{- Sprachliches und fachliches Lernen}

Die in der Veranstaltung vermittelten Inhalte basieren auf dem Prinzip, dass die sprachlichen Lernziele des Unterrichts ausgehend von fachlichen Lernzielen, Lerngegenständen und didaktischen Konzepten zu bestimmen sind. Ziel ist, die unterrichtssprachlichen Kompetenzen der Lernenden ausgehend von der Lernausgangslage systematisch $\mathrm{zu}$ entwickeln (Gibbons 2015). Hierfür ist es notwendig, Zeit und Arbeitsphasen für die Erarbeitung, Sicherung und Übung des sprachlichen Lernziels einzuplanen (Tajmel und Hägi-Mead 2017). Damit dies realistisch möglich ist, ist es notwendig, sich in der sprachlichen Förderung auf die rezeptiven und produktiven sprachlichen Anforderungen zu fokussieren, die für den Unterricht speziell im Fach Chemie von besonderer Relevanz sind. Analog zu den fachlichen Lernzielen ist hierbei zu unterscheiden zwischen themenspezifischen sprachlichen Anforderungen (v. a. Wortschatz, der speziell für ein Thema eingeführt wird) und den nachhaltigen sprachlichen Anforderungen (Wortschatz, Sprachmuster, Textsorten, Darstellungsformen), die über die Jahrgangsstufen hinweg immer wiederkehren bzw. ausgebaut werden (Beese, et al. 2014). Letztere sind im Sinne einer Learning Progression über die Jahrgangsstufen hinweg aufzubauen (Roll et al. 2019a) und ermöglichen oftmals weitere Zugänge zum Verständnis naturwissenschaftlicher Erkenntnisgewinnung (Enzenbach et al. 2019), chemischer Basiskonzepte und der Trennung von Stoff- und Teilchenebene.

In der Veranstaltung werden den Studierenden vor diesem Hintergrund charakteristische sprachliche Anforderungen der chemischen Inhalte des Sachunterrichts und des Chemieunterrichts der Sekundarstufe I sowie didaktische Grundlagen zum sprachsensiblen Unterricht vermittelt (Beese und Kirstein 2018; Streller et al. 2019). Sie lernen charakteristische, aber oft unbeachtete sprachliche Anforderungen auf Wortschatzebene kennen. Ein besonderer Fokus wird auf drei solcher Phänomene gelegt: (1) die Herausforderungen von Verben durch ihre Vorsilben (einsetzen, aufsetzen, zusetzen, versetzen) und ihre spezifischen Präpositionen, (2) auf chemietypische Wortbildungsverfahren (Adjektivbildung mit der Endsilbe -haltig, Stoffbezeichnungen durch die Endsilbe -oxid) und auf Wortbildung basierenden Wortfeldern (lösen, Lösung, löslich, Löslichkeit; Reaktionsgleichung, reaktionsfreudig, Nachweisreaktion) sowie (3) die Auseinandersetzung mit Alltags- und Fachbegriffen (sauer, Gehalt). Sie lernen außerdem, weitere sprachliche (z.B. grammatische) Phänomene zu identifizieren, die in einer Unterrichtseinheit von besonderer Bedeutung sind und nachhaltig im Chemieunterricht benötigt werden (z.B. die grammatische Formulierung, um Voraussetzungen für Verbrennungsreaktionen zu formulieren). Das Prinzip der Learning Progression wird am Beispiel der Entwicklung von Satzmustern für die Kernidee „Bei Stoffumwandlungen werden Stoff zu anderen Stoffen“ vom Sachunterricht bis zum Ende der Sekundarstufe I illustriert, außerdem am Beispiel des Versuchsprotokolls. An diesem Beispiel wird auch das Potenzial des sprachbildenden Unterrichts für die Entwicklung von experimenteller Kompetenz und dem Verständnis für das Prinzip naturwissenschaftlicher Erkenntnisgewinnung verdeutlicht (siehe - Tab. 3.3). 
- Tab.3.3 Auszug aus den sprachlichen Merkmalen des Versuchsprotokolls (Abschnitt Beobachtung)

\begin{tabular}{|c|c|c|}
\hline & Primarstufe & Jahrgangsstufe 5-7 \\
\hline Sinn & $\begin{array}{l}\text { Ich kann sehen/riechen/hören/fühlen, } \\
\text { dass ... }\end{array}$ & $\begin{array}{l}\text { Man kann sehen/riechen/hören/fühlen, } \\
\text { dass ... }\end{array}$ \\
\hline $\begin{array}{l}\text { Zeitpunkt der } \\
\text { Beobachtung }\end{array}$ & $\begin{array}{l}\text { Am Anfang } \ldots \text { Am Ende } \ldots \\
\text { Wenn ich ... hineinschütte, dann } \ldots\end{array}$ & $\begin{array}{l}\text { Am Anfang ... Am Ende ... } \\
\text { Wenn man ... hinzugibt, dann ... } \\
\text { Wenn ... [Zeitpunkt], dann ... }\end{array}$ \\
\hline Ort der Beobachtung & $\begin{array}{l}\text { Am Boden des Glases ..., } \\
\text { In der Flüssigkeit ..., }\end{array}$ & $\begin{array}{l}\text { Am Boden des Glases ..., } \\
\text { In der Flüssigkeit ..., }\end{array}$ \\
\hline \multirow[t]{4}{*}{ Art der Veränderung } & Die Farbe ändert sich. Sie wird ... & $\begin{array}{l}\text { Die Farbe ändert sich von } \ldots \text { zu } \ldots \\
\text { Es gibt einen Farbumschlag von } \ldots \text { zu } \ldots\end{array}$ \\
\hline & Es steigen Blasen auf. & $\begin{array}{l}\text { Es steigen Blasen auf. Es bildet sich also } \\
\text { ein Gas. }\end{array}$ \\
\hline & $\begin{array}{l}\text { Am Boden ist nun ein fester Stoff. Er } \\
\text { ist ... }\end{array}$ & $\begin{array}{l}\text { Es bildet sich ein Feststoff. (Er setzt sich } \\
\text { am Boden/an den Rändern ab.) Er ist/hat } \\
\ldots \text {. [Eigenschaften]. }\end{array}$ \\
\hline & Das Glas wird warm. & Die Temperatur steigt. \\
\hline
\end{tabular}

Die Veranstaltung fokussiert hauptsächlich auf die produktiven sprachlichen Kompetenzen (Klinger et al. 2019). Rezeptive sprachliche Kompetenzen werden jedoch am Beispiel der Lesestrategien für Versuchsvorschriften sowie - als Ausblick auf die höheren Jahrgangsstufen - beim Darstellungswechsel von Modell, Formel- und Schriftsprache thematisiert. Außerdem wird das Potenzial der mehrsprachigen Ressourcen in der Klasse für die Erarbeitung neuer Fachinhalte, die Entwicklung von Textsortenbewusstsein sowie für die Auseinandersetzung mit Schülervorstellungen, die durch Alltags- bzw. mehrdeutige Fachbegriffe hervorgerufen werden, behandelt (Beese und Gürsoy 2019).

Im Rahmen der Planung identifizieren und formulieren die Studierenden angemessene sprachliche Lernziele für ihre Lernsequenzen (Tajmel und Hägi-Mead 2017). Hierbei stehen die Studierenden vor einer besonderen Herausforderung: Normalerweise werden sprachliche Lernziele für Unterrichtsreihen geplant (mit zwei bis drei sprachlichen Lernzielen für eine längere Unterrichtsreihe), da die Erarbeitung eines neuen Fachinhalts idealerweise in einer anderen Unterrichtsstunde stattfindet als die Erarbeitung eines neuen sprachlichen Lernziels und die Übungsphasen für dieses sprachliche Lernziel anschlieBend auf mehrere Unterrichtsstunden verteilt sind (Kniffka 2012). Da die Studierenden in ihren Lernsequenzen einen neuen Fachinhalt und gleichzeitig das sprachliche Lernziel erarbeiten, sichern und üben lassen sollen, muss das sprachliche Lernziel sehr eng gefasst sein, um eine kognitive Überforderung zu verhindern. So kann ein vom Umfang angemessenes sprachliches Lernziel die Wortbildung mit -haltig sein, Verben der Versuchsdurchführung und ihre Vorsilben, die Verben der Zustandsänderungen, die Formulierung von Bedingungen, einzelne Satzmuster zu einer neu entwickelten Kernidee oder zu einem Abschnitt des Versuchsprotokolls, die Reflektion eines Alltags- und Fachbegriffs wie Stoff, Wasser usw. Diese reduzierte Form ist allerdings ausreichend, damit die Studierenden das Prinzip des sprachsensiblen Fachunterrichts anwenden lernen können. 


\subsubsection{Durchführung und Reflexion von Unterricht}

Die Studierenden entwickeln in Kleingruppen je eine Lernsequenz für die Primarstufe (Jahrgangsstufe 3 oder 4) und die Sekundarstufe I (Jahrgangsstufe 6 oder 7), wobei sie von den Lehrenden hinsichtlich der in Abschn. 3.4.2 genannten Schwerpunkte angeleitet und unterstützt werden. In jeder Kleingruppe sind sowohl Grundschul- als auch HRSGe-Studierende. Dies hat sich als besonders produktiv für die Gruppenarbeit erwiesen, da die HRSGe-Studierenden i.d. R. mehr chemisches Fachwissen und Experimentideen mitbringen, die Grundschulstudierenden hingegen mehr Erfahrung im kontextorientierten Lernen sowie in der Verzahnung fachlichen und sprachlichen Lernens.

Etwa zwei Wochen vor den Entdeckertagen werden die geplanten Lernsequenzen in der Veranstaltung gemeinsam mit den anderen Kursteilnehmenden und den Lehrenden ein erstes Mal durchgeführt und reflektiert (Erprobung, vgl. - Abb. 3.13). Auf der Grundlage des Feedbacks überarbeiten die Planungsgruppen ihre Lernsequenzen. Zum Ende jedes Semesters finden zwei Entdeckertage statt. An dem ersten besucht eine Grundschulkasse, am zweiten eine Gesamtschulklasse die Universität. Die Lernenden stammen vielfach aus sozial schwachen Elternhäusern, verfügen über eher geringe bildungssprachliche Fähigkeiten, dafür jedoch über alltagssprachliche Fähigkeiten in mehreren Sprachen. Einzelne Lernende der Grundschule sind erst seit wenigen Monaten in Deutschland. Bei vielen Studierenden führt die Begegnung mit der Lerngruppe zu einem „Realitätsschock“, nach welchem sie - wie sie in der Evaluation angeben - dem sprachsensiblen und differenzierenden Unterrichten einen deutlich höheren Stellenwert beimessen als vorher.

An den Entdeckertagen wird die Kleingruppe der Studierenden halbiert. Die eine Hälfte unterrichtet eine Gruppe von Lernenden, während die andere Hälfte die Durchführung der Lernsequenz mithilfe eines Reflexionsbogens beobachtet, der insbesondere Transparenz und Erreichen des fachlichen und sprachlichen Lernziels sowie Ausgestaltung und Übergang zwischen den Handlungskettenschritten fokussiert. Anschließend wird getauscht. Nun führt die andere Hälfte der Studierenden die Lernsequenz mit einer anderen Schülergruppe durch. Zusätzlich werden die Studierenden bei einer Durchführung videographiert.

Nach den Entdeckertagen wird die Durchführung der Lernsequenzen in zwei Sitzungen videobasiert reflektiert. In der ersten stehen die Qualitätsmerkmale von Unterricht im Fokus der Reflektion, in der zweiten die Verzahnung sprachlichen und fachlichen

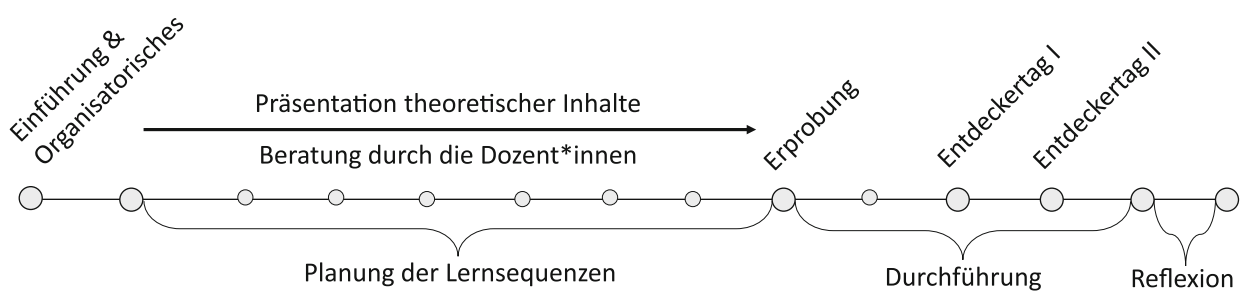

- Abb. 3.13 Struktur der Lehrveranstaltung „Kontextorientierter Sach- und Chemieunterricht“ 
Lernens. Beide werden sowohl auf der Ebene von Stundenstruktur und Arbeitsmaterial als auch auf der Ebene der Unterrichtsinteraktion betrachtet. Die Studierenden erhalten hierfür ausgewählte Videosequenzen, die sie zunächst in Eigenarbeit reflektieren. Anschließend wählen sie besonders gelungene und verbesserungswürdige Sequenzen aus, die sie mit den übrigen Studierenden gemeinsam reflektieren.

Die Modulabschlussleistung besteht in der semesterbegleitenden Verschriftlichung des gesamten Prozesses, angefangen bei der Planung der Lernsequenzen bis zur Reflexion im Sinne eines prozessbegleitenden Portfolios.

\subsubsection{Fazit und Ausblick}

Die regelmäßige Evaluation der Veranstaltung am Ende jedes Semesters, in der der allergrößte Teil der Studierenden die Veranstaltung als (sehr) gewinnbringend bewertet, bestätigt die Lehrenden darin, dass das Grundkonzept das Richtige ist. Insbesondere die Möglichkeit der praktischen Umsetzung und die damit verbundene Konfrontation mit Heterogenität sowie die Möglichkeit zur Videoreflexion werden positiv hervorgehoben. Auch die Verzahnung von sprachlichem und fachlichem Lernen wird von den Studierenden geschätzt, ebenso die gemeinsame Veranstaltung, die Einblicke in die jeweils andere Schulform vermittelt. Kritisiert wird hingegen immer wieder die Diskrepanz zwischen der Hervorhebung der Wichtigkeit der korrekten Antizipation der Lernvoraussetzungen der Lernenden sowohl auf sprachlicher wie auch auf fachlicher Seite und der mangelnden Bereitstellung klassenspezifischer Informationen hierzu. Kurzfristig soll dieses Defizit durch die Erstellung von Material abgemildert werden, das den Kenntnisstand der Lernenden in Bezug auf Sprache und Fachwissen näher beschreiben kann. Mittelfristiges Ziel ist es aber, die Lehrkräfte an den Schulen stärker in die Arbeit an und optimalerweise in der Veranstaltung zu integrieren.

Insgesamt ist im Laufe der Zeit eine Lehrveranstaltung in der Lehramtsausbildung entstanden, die eine theoriegeleitete Sicht auf Unterricht mit Erfahrungen aus der Praxis in Beziehung setzt. Besonders für den Aufbau professioneller Kompetenz im Bereich Sprachbildung ist dies von zentraler Bedeutung, um angehende Lehrkräfte bereits in der ersten Phase der Ausbildung auf die komplexen, aber auch bereichernden Herausforderungen und Chancen sprachlicher Diversität im Schulalltag zeitgemäß und effektiv vorzubereiten.

\subsection{Ausblick}

Die in diesem Buch vorgestellten Konzepte und Materialien für eine sprachbewusste und gleichsam fachbezogene Hochschullehre in der Lehramtsausbildung Mathematik bilden einerseits den Mittelpunkt der Kooperation des Autorenteams, sie stehen andererseits im Zusammenhang mit weiteren gemeinsamen Aktivitäten. Wir möchten daher abschließend einen größeren Rahmen aufspannen, der auch Anregungen für weitere interdisziplinäre Kooperationen geben soll. 


\subsubsection{Aktivitäten im Rahmen der Kooperation}

Eine für die sprachbewusste Hochschullehre in mehrfacher Hinsicht wichtige Schnittstelle bildet die Zusammenarbeit mit Schulen. So stand am Beginn der hier vorgestellten interdisziplinären Kooperation die Beratungsanfrage eines Gymnasiums mit dem Ziel, den Mathematikunterricht sprachbewusster zu gestalten. Wir haben diese Anfrage zum Anlass genommen, um uns am Beispiel eines klar abgegrenzten Themengebiets Wahrscheinlichkeitsrechnung in der 10. Klasse - zunächst über zentrale Inhalte unserer Disziplinen zu verständigen. Als besonders hilfreich erwies sich dabei die gemeinsame, mehrperspektivische Analyse von Materialien aus dem Mathematikunterricht, die uns die Lehrkräfte zur Verfügung stellten (z. B. Aufgaben aus dem verwendeten Lehrwerk, Schülerlösungen zu Klausuren und Beobachtungsdaten aus dem Unterricht). Über die exemplarische Diskussion dieser Materialien kristallisierte sich sukzessive heraus, welche didaktischen Prinzipien und theoretischen Konzepte für die jeweilige Disziplin von Bedeutung sind und wo sich Synergien zeigen, die im besten Fall Sprachbildung als selbstverständlichen Bestandteil des Mathematikunterrichts erkennbar werden lassen. Die Erkenntnisse bildeten die Grundlage für die wissenschaftliche Begleitung der Schule, die zum einen Impulse für die unterrichtliche Entwicklung und die gemeinsame Arbeit an Lehrmaterialien umfasste, zum anderen eine Masterarbeit zur sprachlichen Unterstützung neu zugewanderter Schüler im Mathematikunterricht (Schulze Osthoff 2017) ermöglichte.

Ein zweites Beispiel für den engen Austausch mit einer Schule ist die SchlaU-Schule in München, mit der wir über einen längeren Zeitraum zusammengearbeitet haben. Die Schule ermöglicht geflüchteten Jugendlichen im Alter zwischen 16 und 21 Jahren schulanalogen Unterricht, bereitet sie auf den Ersten allgemeinbildenden Schulabschluss (ESA) vor und begleitet ihren Übergang in die Berufsausbildung. In der zugehörigen SchlaU-Werkstatt für Migrationspädagogik werden zielgruppenadäquate Lehr-LernMaterialien für Deutsch als Zweitsprache und sprachsensiblen Fachunterricht entwickelt und publiziert. Wir begleiteten die Konzeption von Lehr-Lern-Materialien für das Fach Mathematik (z. B. SchlaU-Werkstatt für Migrationspädagogik 2020) mit Workshops, durch die Kommentierung von Materialentwürfen aus mathematikdidaktischer und sprachbildender Sicht sowie durch die Einbindung von Studierenden, die im Rahmen von Bachelor- bzw. Masterarbeiten (Scharnofske 2019, Schmitt 2019) ausgewählte Materialien erprobten, adaptierten und Rückmeldungen dazu gaben. So erweist sich die Zusammenarbeit auch unter dem Forschungsaspekt als weiterführend.

\section{Konzeptbildung durch Studierende in der Schule begleiten}

Viele Studierende erleben in Praxisphasen des Studiums, dass die Fachgruppen Mathematik an den Schulen sich fragen, wie sich fachdidaktische Konzepte für einen sprachbewussten Mathematikunterricht für das eigene bzw. in der Fachgruppe abgestimmte unterrichtliche Handeln nutzen lassen - so etwa die in Abschn. 2.3 dargestellten Aufgabenvariationen als Beispiele für die Adressierung von Sprachbildung durch die Nutzung unterschiedlicher Repräsentationsmittel im Mathematikunterricht. Diskutiert werden dabei ebenso 
fachbezogene Anlässe, um etwa die Arbeit an Aufgaben und Aufgabenvariationen für die konzeptionelle Arbeit in fruchtbarer Weise zu nutzen.

Viele Schulen haben mittlerweile Konzepte zur Sprachbildung erstellt. Ein Mangel dieser Konzepte wird von vielen Kolleginnen und Kollegen häufig aber noch darin gesehen, dass ihnen der konkrete fachspezifische Übertrag schwerfällt bzw. die Ausführungen häufig als sehr allgemein empfunden werden und dadurch nur bedingt für den eigenen Unterricht adaptierbar sind. Daher besteht ein besonderes Potential darin, allgemeine Konzepte zur Sprachbildung um jeweils fachspezifische Teile zu ergänzen. Einen Beitrag kann in diesem Zusammenhang die Entwicklung eines abgestimmten Konzepts zur Sprachbildung im Fachunterricht leisten. Ein solches Konzept wollte eine Fachgruppe Mathematik an einer weiterführenden Schule für sich erarbeiten, bei der eine Studierende ihr Praxissemester absolvierte. Die Studierende verknüpfte die Arbeit an diesem Konzept mit ihrem Studienprojekt, das sich thematisch der Begleitung des Entwicklungsprozesses widmete. Eine vertiefende Betrachtung konnte im Anschluss im Rahmen der Masterarbeit im Fach Mathematik vorgenommen werden. Ausgangspunkt für die Entwicklung eines solchen Konzeptes bildete in dem hier vorgestellten Beispiel die Arbeit an und Variation von Aufgaben.

Anlass war zunächst der große Bedarf an Sprachbildung im Unterricht generell, bedingt durch eine sprachlich sehr heterogene Schülerschaft. Im Rahmen der Arbeit mit der Fachgruppe wurde zunächst der oben beschriebene inhaltliche Schwerpunkt gesetzt: Die Fachgruppe entschied sich, Darstellungsvernetzung als Mittel der Sprachbildung im Mathematikunterricht gezielt zu nutzen. Da das im Unterricht genutzte Mathematikbuch Darstellungswechsel eher sparsam einsetzte, hat die Fachgruppe sich dafür entschieden, zunächst einige Beispielaufgaben zu unterschiedlichen Themen unterschiedlicher Jahrgänge auszuwählen und zu diskutieren. Vor diesem Hintergrund wurden dann gemeinsame Aufgabenvariationen mit dem Ziel vorgenommen, die Aufgaben im Sinne der Sprachbildung durch gezielte Darstellungsvernetzung zu verändern. Um vor diesem Hintergrund den Prozess der Konzepterstellung gezielt anzustoßen, wurden folgende Leitfragen entwickelt, die von der Studierenden im Rahmen der Arbeit qualitativ ausgewertet wurden:

- Welche Bedarfe an Sprachbildung im Fach bestehen aus Sicht der Lehrkräfte bzw. des jeweiligen Fachunterrichts?

- Wie wurde bei der Variation der Aufgaben vorgegangen?

- Worauf muss bei der Aufgabenvariation geachtet werden?

- Welche Kriterien sind bei der Formulierung eines Konzeptes zur Sprachbildung im Fach notwendig?

Auf diese Weise wurden von den Lehrkräften zunächst - ausgehend vom genutzten Mathematikbuch - Aufgabenvariationen entwickelt, anhand derer der Prozess der Erstellung eines solchen Konzeptes zur fachbezogenen Sprachbildung initiiert wurde. Die Studierende hat im Rahmen dieses Prozesses gleichzeitig einen Beitrag zur Innovation von unterrichtlichen Entwicklungsprozessen geleistet und konnte wertvolle Einsichten in die konzeptionelle Arbeit von Lehrkräften gewinnen.

Einen ganz anderen Aspekt unserer Kooperation stellt die Weiterentwicklung der gemeinsamen Lehrveranstaltungen dar. Für Studierende bedeutet das Lernen und Forschen unter Berücksichtigung zweier Disziplinen oft eine große Herausforderung: Sie 
müssen die Doppelperspektive von Fach und Sprache einnehmen, ggf. auch in besonders beanspruchenden Kontexten wie dem forschenden Lernen in Praxisphasen oder bei der Anfertigung einer Abschlussarbeit. Um den Studierenden eine optimale Verknüpfung von fach- und sprachdidaktischen Elementen in der Lehramtsausbildung zu bieten, entwickeln wir die Lehre kontinuierlich weiter. Zentrale Fragen hierbei sind u. a.:

- Wie kann das Thema Sprachbildung in fachdidaktischen Veranstaltungen selbst durchgängig umgesetzt werden?

- Wie kann eine Ausgewogenheit von fachlichen/fachdidaktischen und sprachlichen/ sprachdidaktischen Inhalten hergestellt werden?

- Welche Materialien und Übungen eignen sich, um die Studierenden insbesondere beim forschenden Lernen zu unterstützen?

- Wie kann eine enge Verknüpfung von universitären Ausbildungsinhalten und schulpraktischen Erfahrungen hergestellt werden?

- Welche Inhalte, Forschungsmethoden und Formen der kollegialen Zusammenarbeit können die Studierenden langfristig in ihren Schulalltag integrieren?

Dafür erweisen sich regelmäßige studentische Rückmeldungen während des Seminars und im Rückblick als hilfreich, wie in $>$ Abschn. 3.1 dargelegt. Ein Forschungsdesiderat bildet die längerfristige systematische Begleitung ehemaliger Studierender in ihrer schulischen Praxis.

Die Frage, wie sich Sprachbildung in allen Fächern und für spezifische Lernendengruppen bestmöglich umsetzen lässt und wie eine entsprechende Vorbereitung angehender Lehrkräfte aussehen kann, ist international weiterhin von großer Bedeutung. Daher spielen selbstverständlich auch der intensive Austausch und die Vernetzung mit Forschenden aus dem In- und Ausland sowie entsprechende Publikationstätigkeiten eine wichtige Rolle.

\subsubsection{Sprachbildung in der Hochschule: Ansatzpunkte zur Vernetzung}

Abschließend seien an dieser Stelle einige Anregungen genannt, um eine interdisziplinäre Vernetzung auf Hochschulebene zu initiieren. Um nicht missverstanden zu werden: Auch wenn die hier beschriebenen Konzepte in einer Kooperation von Mathematikdidaktik und Sprachdidaktik entstanden sind, so lässt sich vieles natürlich auch ohne eine solche interdisziplinäre Zusammenarbeit im eigenen Fach auf den Weg bringen. Die hier beschriebenen Konzepte und Ideen eignen sich aus unserer Sicht insbesondere auch als Beispiele und Anregungen für die Adaption in der eigenen Lehre (im Fach).

Gleichwohl kann eine bewusste Kooperation zwischen Sprachbildung und Fach hilfreiche und wichtige Impulse für die Weiterentwicklung der eigenen Lehre und darüber hinaus liefern. Zu Beginn einer solchen Kooperation kann es sinnvoll sein, sich über zentrale Konzepte auszutauschen, die die jeweilige Disziplin kennzeichnen. Dafür ist es lohnenswert, zunächst einige Themen zusammenzustellen, die regulärer Bestandteil der fachinhaltlichen, fachdidaktischen bzw. DaZ-/sprachdidaktischen Veranstaltungen sind und die eventuell schon Anknüpfungspunkte zur jeweils anderen Disziplin eröffnen könnten. 
- Beispiel Fachdidaktik Mathematik: Vorstellungsorientierung und kognitive Aktivierung; Begriffsbildung; Rolle von Darstellungen; prozessbezogene Kompetenzen (,Argumentieren“, „Kommunizieren“), Lernen mit digitalen Werkzeugen usw.

- Beispiel DaZ-/Sprachdidaktik: Linguistische Analyse von Fachtexten und Schülerdokumenten aus dem Fachunterricht; Leitlinien zur Sprachbildung im Fach; Ansätze wie Scaffolding, SIOP, genrepädagogischer Lehr-Lern-Zyklus; Mehrsprachigkeit im Fachunterricht; Sprachvergleich usw.

Im gemeinsamen Austausch - idealerweise anhand konkreter praxisnaher Materialien (z. B. Aufgabenstellungen und Texte aus Lehrwerken, schriftliche Lernendendokumente, von Lehrkräften erstelltes Lern- oder Prüfungsmaterial, Videos aus dem Fachunterricht, Lehrpläne usw.) - lassen sich Aspekte identifizieren, bei denen Gemeinsamkeiten und Anregungen für die Zusammenarbeit sichtbar werden. Für den Einstieg in eine Kooperation kann es sinnvoll sein, einen konkreten Anlass für die Zusammenarbeit zu definieren. Dabei sind natürlich viele Varianten denkbar, zum Beispiel:

- Planung einer gemeinsamen Lehrveranstaltung oder einzelner gemeinsamer Sitzungen

- Entwicklung und Pilotierung von sprachbildenden Unterrichtsmaterialien

- wissenschaftliche Begleitung einer Schule bei der Entwicklung eines sprachbewussten Unterrichtskonzepts

- interdisziplinäre Betreuung von Studierenden im Kontext einer Praxisphase oder bei einer Abschlussarbeit

- Forschung an der Schnittstelle von Fach und Sprache zur Vorbereitung eines Tagungsbeitrags/einer Publikation/eines Projekts

Wir wünschen den Leserinnen und Lesern dieses Buches nicht nur eine anregende Arbeit bei der Weiterentwicklung der eigenen Lehre, sondern auch spannende neue Einsichten durch Kooperationen, die auch den Blick auf das eigene Fach erweitern können.

Open Access Dieses Kapitel wird unter der Creative Commons Namensnennung 4.0 International Lizenz (http://creativecommons.org/licenses/by/4.0/deed.de) veröffentlicht, welche die Nutzung, Vervielfältigung, Bearbeitung, Verbreitung und Wiedergabe in jeglichem Medium und Format erlaubt, sofern Sie den/die ursprünglichen Autor(en) und die Quelle ordnungsgemäß nennen, einen Link zur Creative Commons Lizenz beifügen und angeben, ob Änderungen vorgenommen wurden.

Die in diesem Kapitel enthaltenen Bilder und sonstiges Drittmaterial unterliegen ebenfalls der genannten Creative Commons Lizenz, sofern sich aus der Abbildungslegende nichts anderes ergibt. Sofern das betreffende Material nicht unter der genannten Creative Commons Lizenz steht und die betreffende Handlung nicht nach gesetzlichen Vorschriften erlaubt ist, ist für die oben aufgeführten Weiterverwendungen des Materials die Einwilligung des jeweiligen Rechteinhabers einzuholen.

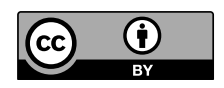

